\title{
A Comprehensive Screening and Identification of Genistin Metabolites in Rats Based on Multiple Metabolite Templates Combined with UHPLC-HRMS Analysis
}

\author{
Yaoyue Liang ${ }^{1}$, Wenjing Zhao ${ }^{1}$, Chenxiao Wang ${ }^{1}$, Zijian Wang ${ }^{2}$, Zhibin Wang ${ }^{2, *}$ \\ and Jiayu Zhang ${ }^{2, *}$ \\ 1 School of Chinese Pharmacy, Beijing University of Chinese Medicine, Beijing 100029, China; \\ lyy2610@163.com (Y.L.); cosyzwj@126.com (W.Z.); 18801381993@163.com (C.W.) \\ 2 Beijing Research Institution of Chinese Medicine, Beijing University of Chinese Medicine, \\ Beijing 100029, China; helloffiresilver@gmail.com \\ * Correspondence: wangzhibin4804@126.com (Z.W.); zhangjiayu@bucm.edu.cn (J.Z.); \\ Tel.: +86-10-6428-7540 (Z.W. \& J.Z.)
}

Received: 29 June 2018; Accepted: 19 July 2018; Published: 26 July 2018

\begin{abstract}
Genistin, an isoflavone belonging to the phytoestrogen family, has been reported to possess various therapeutic effects. In the present study, the genistin metabolites in rats were investigated by UHPLC-LTQ-Orbitrap mass spectrometer in both positive and negative ion modes. Firstly, the data sets were obtained based on data-dependent acquisition method and then 10 metabolite templates were established based on the previous reports. Then diagnostic product ions (DPIs) and neutral loss fragments (NLFs) were proposed to efficiently screen and ascertain the major-to-trace genistin metabolites. Meanwhile, the calculated $C \log P$ values were used to identify the positional isomers with different retention times. Consequently, a total of 64 metabolites, including prototype drug, were positively or putatively characterized. Among them, 40 metabolites were found according to the templates of genistin and genistein, which was the same as the previous research method. After using other metabolite templates, 24 metabolites were added. The results demonstrated that genistin mainly underwent methylation, hydrogenation, hydroxylation, glucosylation, glucuronidation, sulfonation, acetylation, ring-cleavage and their composite reactions in vivo biotransformation. In conclusion, the research not only revealed the genistein metabolites and metabolic pathways in vivo comprehensively, but also proposed a method based on multiple metabolite templates to screen and identify metabolites of other natural compounds.
\end{abstract}

Keywords: genistin; UHPLC-LTQ-Orbitrap mass spectrometer; multiple metabolite templates; metabolic profiling

\section{Introduction}

Recently, isoflavones have received much attention due to their nutritional and potential health benefits. They are non-steroidal phytoestrogenic and antioxidative polyphenolic molecules, which have the possibilities to protect against hormone-dependent diseases, such as prostate cancer, breast cancer, menopausal symptoms, cardiovascular disease and osteoporosis [1-3]. Genistin, as one of the common isoflavones, has a similar structure with phytoestrogens and belongs to the annual plant of Fabaceae family especially Glycine max (L.) MERR. It possesses various therapeutic effects, including anti-inflammatory, anticancer activities, cardioprotective effects, antioxygenation, etc., through pharmacological studies [4-7]. For example, it was found that genistin could 
enhance the expression of acetylcholinesterase (AChE) related enzymes and related proteins in cultured rat osteoblasts. Therefore, it was speculated that genistin could improve osteoporosis [8]. Compared with other drugs, genistin has many advantages, such as abundant sources, stable nature, low toxicity and side effects. Although there is no clear evidence that the ingestion of genistin is harmful, genistin (i.e., genistein-7-glucoside) can metabolize to produce genistein after entering the body. The genotoxicity and potential adverse effects (cell growth inhibition, apoptosis, topoisomerase inhibition, and DNA damages) of genistein were reported in vitro as well as in experimental animals (e.g., rats) [9-13]. Genistin produces metabolic products of different biological effects through metabolic process in vivo, and the metabolic level of different organisms is different. However, it is still ambiguous that what the material basis is responsible for the effects of genistin. But it is certain that metabolites play an important role. Therefore, it is necessary to study the metabolism of genistin, which helps to explore its effects on cardiovascular and cerebrovascular diseases, tumor and other diseases. And it is of great significance to understand the biological activity of isoflavones and explore its development and utilization.

Contemporarily, ultra-high performance liquid chromatography (UHPLC) coupled with mass spectrometry (MS) has been widely used in drug metabolism analysis [14-16]. UHPLC has a powerful separation capability, which is currently one of the most effective tools for the complex components separation. MS owns the properties of high speed, sensitivity and selectivity, during which the high resolution (HR) and multistage mass spectrometry technology have been comprehensively used for online structural analysis and quantitative detection of known and unknown components. In recent years, with the continuous development of Orbitrap technology, Orbitrap mass spectrometer is widely used in pharmaceutical analysis due to the HR detection through combining with the linear ion trap [17-19]. Nowadays, UHPLC-LTQ-Orbitrap mass spectrometer has been widely applied in metabolism studies [20-24].

To date, there are dozens of reports concerned with the techniques of dealing the dataset to extract the metabolite information. These techniques include the adoption of mass defect filters, isotope pattern filters, and background subtraction and so on [25]. Generally, the parent drug is selected as the template to predict metabolites according to the common metabolic pathways. The obvious limitation is that many drug metabolites are yielded through the composite reactions. In the previous studies of the metabolites of genistin and the corresponding aglycone (genistein), the number of the detected metabolites was small [26-29]. This greatly hinders the discussion of the material basis for the efficacy of genistin after entering the body. The characteristic of our study is that according to the literature reports and the results of pre experiments, the multiple metabolite templates are established to assist with data processing. In combination of diagnostic product ions (DPIs), neutral loss fragments (NLFs) and calculated $C \log P$ values, the potential metabolites of genistin were proposed in this study. As far as we know, it was the first time to comprehensively investigate the metabolism of genistin in vivo.

\section{Results and Discussion}

\subsection{The Establishment of Analytical Strategy}

In this study, we established a comprehensive and effective strategy to discover and identify genistin metabolites by using UHPLC-HRMS. At the beginning, HR-ESI-MS ${ }^{1}$ analysis was performed on the HRMS instrument with a resolution of 30,000. Meanwhile ESI-MS ${ }^{n}$ data sets were got both in negative and positive ion modes based on data-dependent acquisition method. Secondly, the metabolite templates were established according to the literature reports and the pre experiment results. Then DPIs and NLFs, which were proposed by the mass fragmentation behaviors of reference standards, were applied to efficiently confirm and identify the genistin metabolites. After that, according to different retention times, positional isomers were distinguished by using the corresponding Clog $P$ values calculated by ChemBioDraw Ultra 14.0 (PerkinElmer, Waltham, MA, USA). Depending on the information obtained above, all metabolites were identified positively or 
putatively. Finally, grounded on metabolites data and references, the metabolic pathway of genistin was proposed. Through the whole experiment, we had summed up a schematic diagram, as shown in Figure 1, which was an analytical strategy for detecting and identifying genistin metabolites.

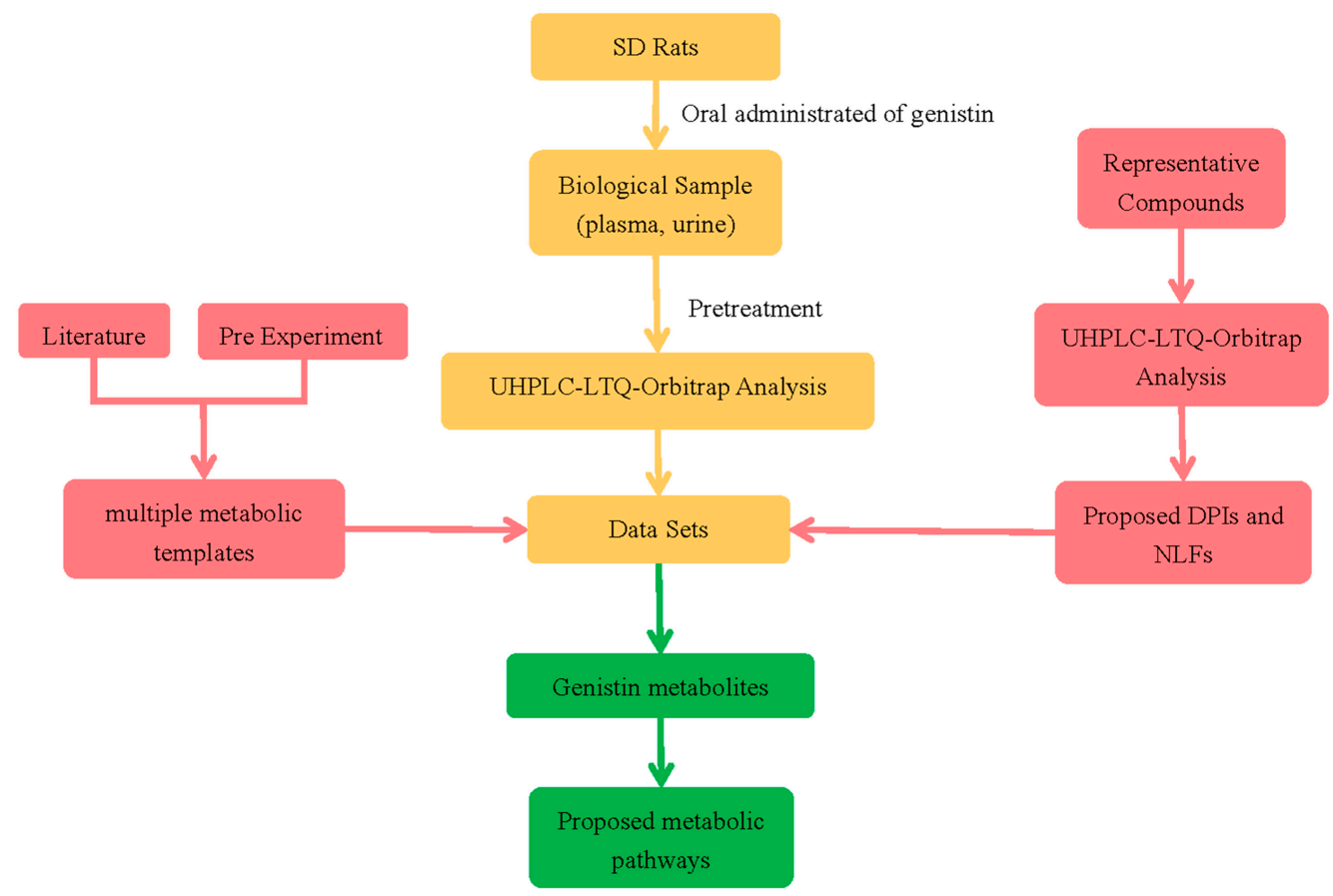

Figure 1. The summary diagram of analytical strategy for detection and identification of genistin metabolites.

\subsection{The Establishment of Multiple Metabolite Templates Screening Method}

Ten metabolite templates, genistin, genistein, daidzin, daidzein, puerarin, dihydrogenistein, tetrahydrogenistein, dihydrodaidzein, equol, and $O$-desmethylangolensin, were selected as the screening templates for genistin metabolites. Of course, based on this method, the metabolites selected from different templates would be the same. Combined with the retention time, DPIs, and NLFs, the compounds with the same structure need to be deleted. Based on this efficient method, some uncommon compound metabolites could be much more comprehensively detected.

\subsection{Establishment of Diagnostic Product Ions Basing on Fragmentation Behaviors of Genistin and Its Homologues}

In order to obtain an extensive fragmentation behavior of genistin and the other four homologues (genistein, daidzin, daidzein, and puerarin) (Figure 2), the mixed standard solution was comprehensively analyzed by using UHPLC-LTQ-Orbitrap MS. It can provide the useful information, such as DPIs and characteristic fragmentation patterns, which were utilized for deducting the structures of related metabolites. Taking genistin as an example, it showed a protonated $[\mathbf{M}+\mathbf{H}]^{+}$ion at $m / z$ $433.1110\left(\mathrm{C}_{21} \mathrm{H}_{21} \mathrm{O}_{10},-4.39 \mathrm{ppm}\right)$ with the retention time of $4.59 \mathrm{~min}$ in the ESI-MS experiment. It produced the base peak ion at $m / z 271[\mathrm{M}+\mathrm{H}-162]^{+}$via the loss of glucose moiety in the ESI-MS ${ }^{2}$ spectrum. In the ESI-MS ${ }^{3}$ experiment, $m / z 153$ was yielded as the base peak via Retro-Diels-Alder (RDA) fragmentation by losing $\mathrm{C}_{8} \mathrm{H}_{6} \mathrm{O}$. Meanwhile, the other characteristic ions at $m / z 215(81 \%)$, $243(59 \%), 253(34 \%), 149(30 \%), 145(16 \%)$, and $225(11 \%)$ were yielded by losing $2 \mathrm{CO}, \mathrm{CO}, \mathrm{H}_{2} \mathrm{O}$, $\mathrm{C}_{7} \mathrm{H}_{6} \mathrm{O}_{2}, \mathrm{C}_{6} \mathrm{H}_{6} \mathrm{O}_{3}$, and $\mathrm{CO}+\mathrm{H}_{2} \mathrm{O}$, as shown in Figure 3. For genistein with retention time of $8.00 \mathrm{~min}$, the MS ${ }^{2}$ ions at $m / z 153(100 \%), 215(79 \%), 243(62 \%), 253(34 \%), 149(29 \%), 145(18 \%)$, and $225(10 \%)$ were the same as the ESI-MS ${ }^{3}$ ions of genistin, which could be attributed to the fact that genistin is the 
glucoside of genistein. Meanwhile, daidzin and daidzein were eluted at 3.86 and 6.28 min, respectively. In the $\mathrm{MS}^{3}$ spectrum of daidzin and $\mathrm{MS}^{2}$ spectrum of daidzein, the most abundant fragment ion at $m / z 199$ was produced due to the loss of 2CO. Moreover, several major fragment ions at $m / z 137$, $m / z 227, m / z 237$, and $m / z 145$ were also observed due to the respective loss of $\mathrm{C}_{8} \mathrm{H}_{6} \mathrm{O}, \mathrm{CO}, \mathrm{H}_{2} \mathrm{O}$, and $\mathrm{C}_{6} \mathrm{H}_{6} \mathrm{O}_{2}$, which indicated that they owned the similar cracking behavior with genistin [26].

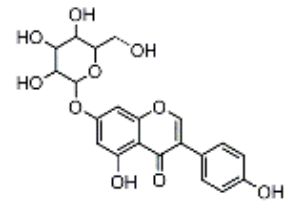

Genistin<smiles>O=C(c1ccc(O)cc1)c1coc2cc(O)cc(O)c12</smiles>

Genistein

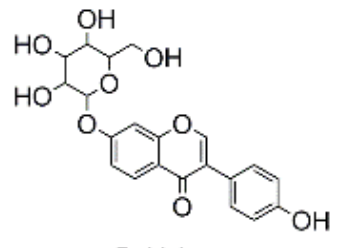

Daidzin<smiles>O=c1c(-c2ccc(O)cc2)coc2cc(O)ccc12</smiles>

Daidzein

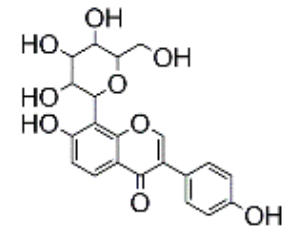

Puerain

Figure 2. The chemical structures of the five reference standards.

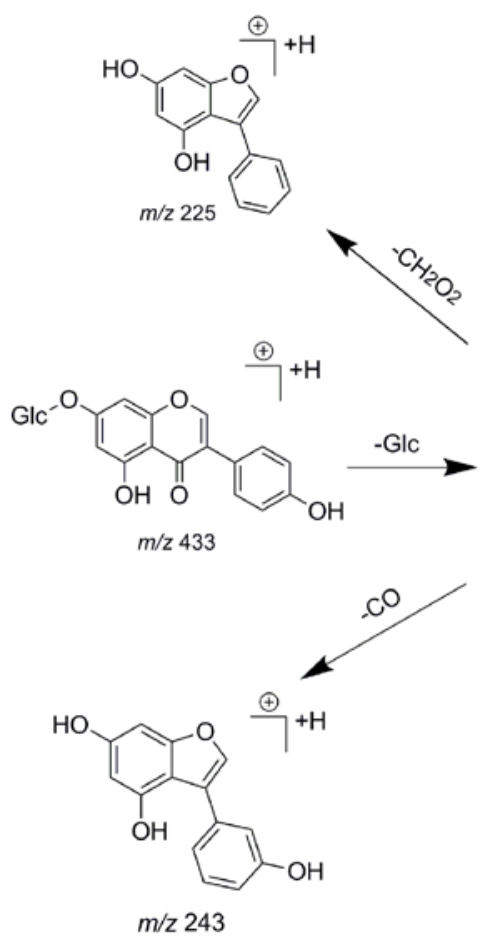<smiles>Oc1cc(O)c2ccoc2c1</smiles>

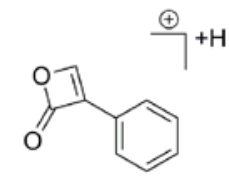
$m / z 145$

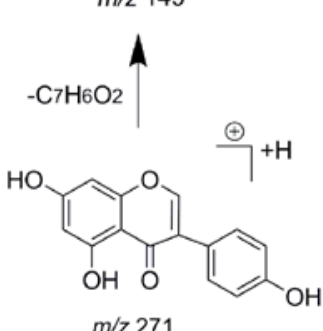

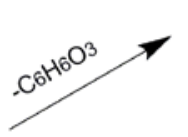<smiles>CCC</smiles><smiles>O=c1c(-c2ccccc2)coc2cc(O)cc(O)c12</smiles>

$m / z 253$

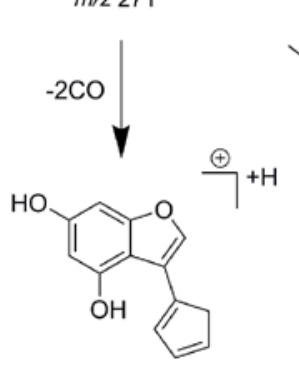

$m / z 215$

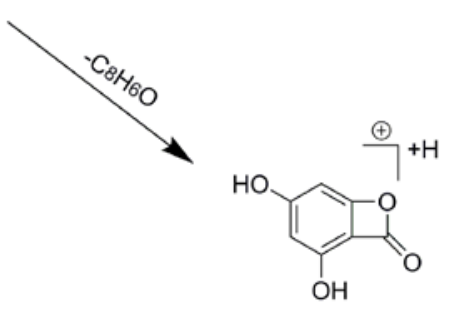

$m / 2153$

Figure 3. The fragmentation behavior of genistin in positive ion mode.

In addition, it was necessary to note that $m / z 269$ and $m / z 268$ were produced in the ESI-MS ${ }^{2}$ of genistin in negative ion mode under the lower collision energies. It showed the loss of a $\mathrm{C}_{6} \mathrm{H}_{10} \mathrm{O}_{5}$ (162 Da) moiety and the corresponding pure scission process by losing $\mathrm{C}_{6} \mathrm{H}_{11} \mathrm{O}_{5}(163 \mathrm{Da})$ moiety, which resulted in the formation of the aglycone and free radical aglycone product ions [30]. Some of the main characteristic ions were generated by rearrangement and scission. For example, $m / z 267(100 \%)$, $239(81 \%)$, and $223(56 \%)$ were occurred in the ESI-MS ${ }^{3}$ spectrum. The residues in the ESI-MS ${ }^{3}$ of 
genistin were similar to those in the ESI-MS² spectrum of genistein as showed in Figure 4 , which could facilitate the structural elucidation of the genistin metabolites in vivo. Besides, the fragmentation pathways of daidzin and daidzein were also similar to those of genistin [31].

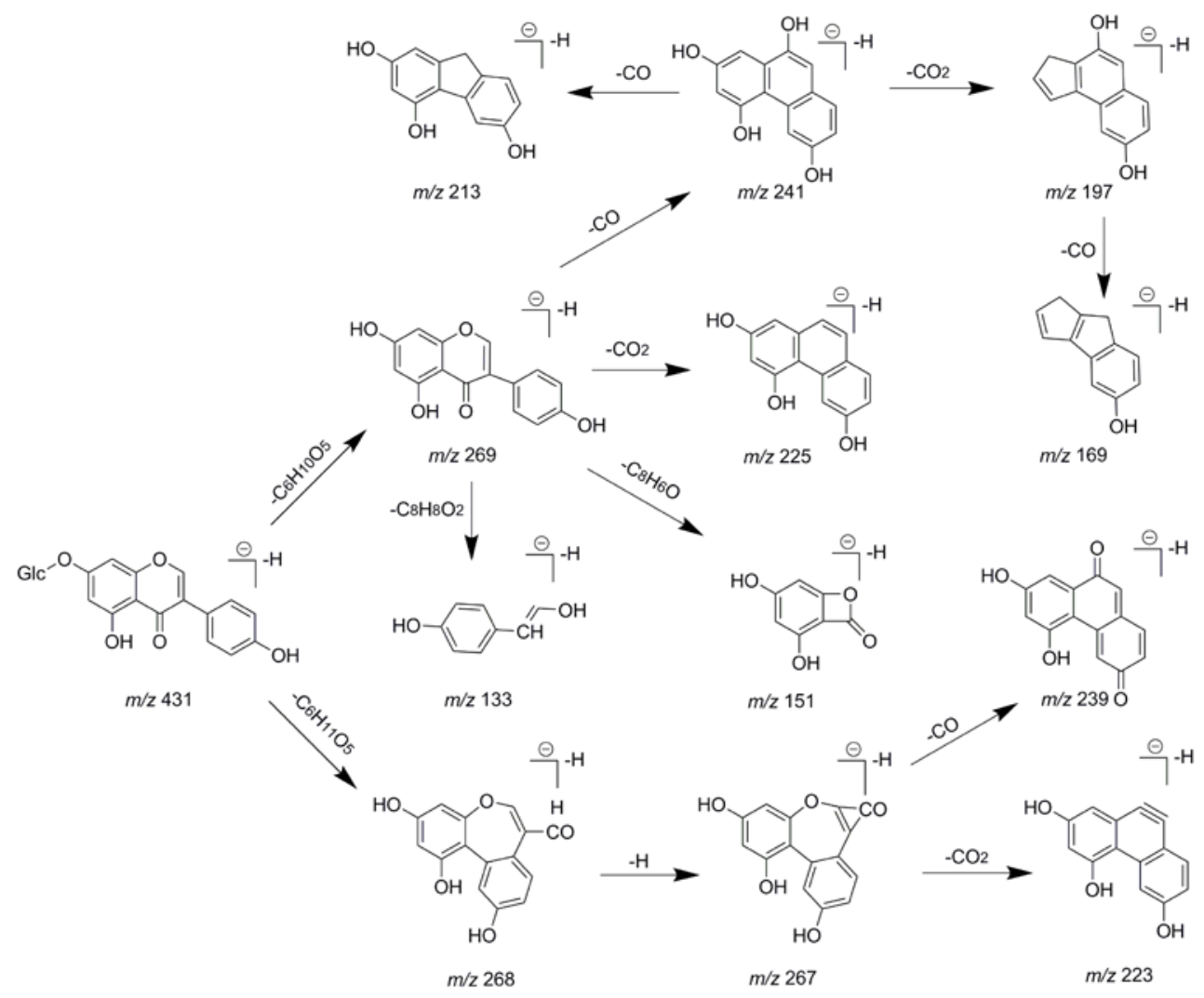

Figure 4. The fragment behaviors of genistin in negative ion mode.

Unlike the above four reference standards, puerarin, attributed to C-glycoside, possessed its own unique cleavage mode. Taking puerarin in positive ion mode as an example, it yielded the protonated molecule $[\mathrm{M}+\mathrm{H}]^{+}$ion at $m / z 417.1160\left(\mathrm{C}_{21} \mathrm{H}_{21} \mathrm{O}_{9},-4.89 \mathrm{ppm}\right)$ in the ESI-MS ${ }^{1}$ spectrum. In the ESI-MS ${ }^{2}$ spectrum, some fragmentation ions, occurred on heteroside moiety, were generated, including $m / z 399(100 \%), m / z 351(53 \%), m / z 381(36 \%), m / z 297(31 \%), m / z 363$ (14\%), and $m / z 321$ $(12 \%)$ by NLFs of $\mathrm{H}_{2} \mathrm{O}, 2 \mathrm{H}_{2} \mathrm{O}+\mathrm{CH}_{2} \mathrm{O}, 2 \mathrm{H}_{2} \mathrm{O}, \mathrm{C}_{4} \mathrm{H}_{6} \mathrm{O}_{3}, 3 \mathrm{H}_{2} \mathrm{O}$, and $2 \mathrm{H}_{2} \mathrm{O}+2 \mathrm{CH}_{2} \mathrm{O}$. The fragmentation pathways of puerarin in positive and negative ion modes are proposed in Figure 5 [32,33].

To facilitate the structural illustration of genistin metabolites in the complex matrix, the DPIs were applied to distinguish genistin metabolites in this study [25]. The homologues possessed the similar fragmentation behaviors, which meant that there would be similar DPIs and regular NLFs for providing adequate evidences for elucidating the metabolites. With the biological reactions in vivo, the DPIs might change accordingly. For instance, in positive ion mode, the aglycone moiety of genistin was easily lost in ESI-MS analysis, and the DPI at $m / z 271\left[\mathrm{M}+\mathrm{H}-\mathrm{C}_{6} \mathrm{H}_{10} \mathrm{O}_{5}\right]^{+}$would be produced in ESI-MS 2 spectrum. If some different kinds of biological reactions occurred on the original drug (not on the aglycone moiety), new DPIs resulted from newly generated metabolites at $m / z 271+X$ (X: mass weight of substituent groups, such as $14,16,80,162$, etc.) giving information about the type of the substituent groups. Besides, the DPI at $m / z 153$ was generated due to RDA rearrangement which occurred on the 1,4-position of C-ring of genistin in positive ion mode. It would give the information whether bio-reactions occurred on the A-ring or not. To point out, NLFs also provided tremendous help for efficiently elucidating the structures of unknown metabolites. For instance, the successive losses of $28 \mathrm{Da}(\mathrm{CO})$ eliminated in the ESI-MS ${ }^{\mathrm{n}}$ spectra of genistin and the NLF of $44 \mathrm{Da}\left(\mathrm{CO}_{2}\right)$ was only found 
in negative ion mode of genistin, which could also offer much information to identify these metabolites. Although the metabolites were difficult to identify because of the absence of corresponding reference standards, the DPIs coupled with NLFs were calculated to clarify these complex metabolites.
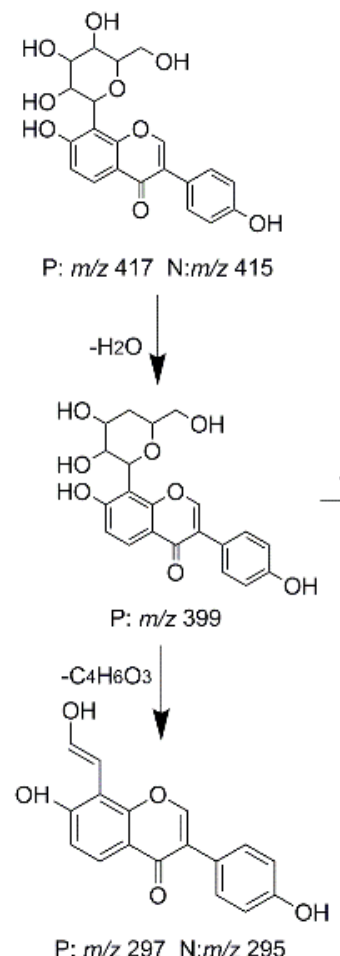
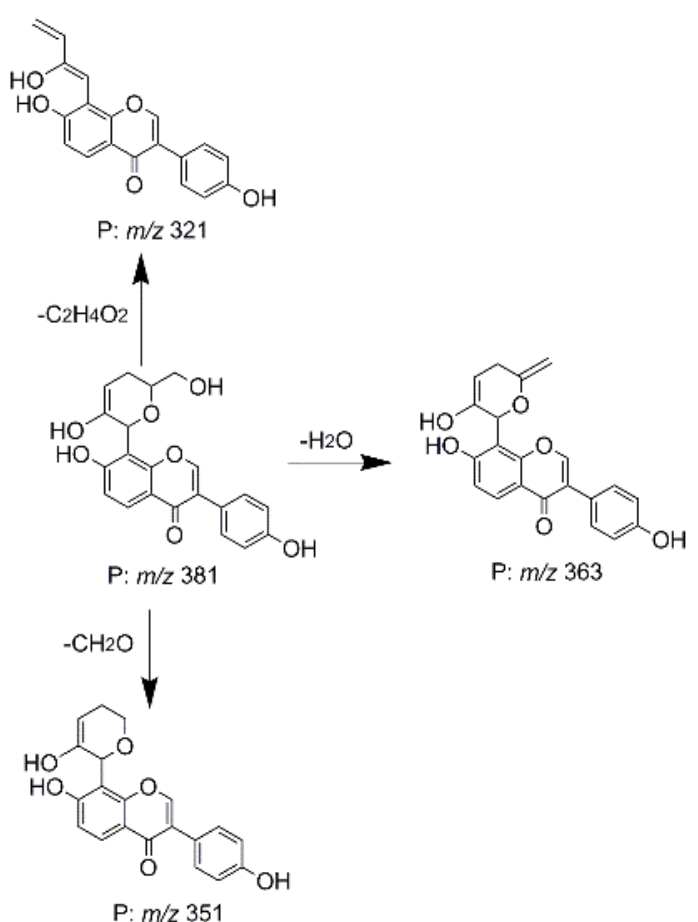

Figure 5. The fragmentation patterns of puerarin ( $\mathrm{P}$ for the positive ion mode and $\mathrm{N}$ for the negative ion mode).

\subsection{Identification of Genistin Metabolites in Rats}

The total ion chromatograms (TICs) of urine and plasma samples from the rats after oral administration of genistin were obtained by using UHPLC-LTQ-Orbitrap mass spectrometry. After comparing the drug-obtained samples with the corresponding control ones, 64 metabolites were detected in both positive and negative ion modes, among which 39 metabolites in positive ion mode and 43 metabolites in negative ion mode were identified in urine, while six metabolites in positive ion mode and eight metabolites in negative ion mode were identified in plasma. The high-resolution extracted ion chromatograms (HREICs) of related metabolites were showed in Figure 6 and the chromatographic retention times and some other concerned data were summarized in Table 1.

\subsubsection{Identification of Metabolites with the Templates of Genistin and Genistein}

Based on the templates of genistin and its aglycone, genistein, 40 compounds were identified, labeled from M0 to M39.

M0 and M12, which possessed the same deprotonated $[\mathrm{M}-\mathrm{H}]^{-}$ions at $m / z 431.0972\left(\mathrm{C}_{21} \mathrm{H}_{19} \mathrm{O}_{10}\right.$, error within $\pm 3.50 \mathrm{ppm}$ ), were eluted at 4.64 and $5.01 \mathrm{~min}$, respectively. By comparing the full-scan $\mathrm{MS} / \mathrm{MS}^{\mathrm{n}}$ spectra and retention time with the reference standard, M0 was unequivocally deduced to be the parent drug. The accurate mass weight and major product ions $(m / z 268, m / z 269, m / z 223$, $m / z$ 224, and $m / z 267$, etc.) of $\mathbf{M 1 2}$ were coincident with those of M0, indicating that it could be deduced as a genistin isomer. 
Table 1. Summary of genistin metabolites in rat urine and plasma by HPLC-LTQ-Orb.

\begin{tabular}{|c|c|c|c|c|c|c|c|c|c|c|c|}
\hline Peak & $\begin{array}{l}\text { Ion } \\
\text { Mode }\end{array}$ & $t_{\mathrm{R}} / \min$ & Formula & $\begin{array}{l}\text { Theoretical } \\
\text { Mass } m / z\end{array}$ & $\begin{array}{l}\text { Experimental } \\
\text { Mass } m / z\end{array}$ & $\begin{array}{r}\text { Error } \\
(\mathrm{ppm})\end{array}$ & MS/MS Fragment Ions & Identification/Reactions & $\begin{array}{l}C \operatorname{Clog} P \\
\text { Value }\end{array}$ & $\mathbf{U}$ & $\mathbf{P}$ \\
\hline \multirow[t]{2}{*}{ M0 } & $\mathrm{P}$ & 4.64 & $\mathrm{C}_{21} \mathrm{H}_{21} \mathrm{O}_{10}$ & 433.1129 & 433.1119 & -2.34 & $\begin{array}{c}\mathrm{MS}^{2}[433]: 271(100) \\
\mathrm{MS}^{3}[271]: 153(100), 215(81), 243(66), \\
\text { 271(34), 253(28) }\end{array}$ & & 0.91 & + & - \\
\hline & $\mathrm{N}$ & 4.64 & $\mathrm{C}_{21} \mathrm{H}_{19} \mathrm{O}_{10}$ & 431.0972 & 431.0985 & 2.73 & $\begin{array}{c}\mathrm{MS}^{2}[431]: 268(100), 269(75) \\
\mathrm{MS}^{3}[268]: 267(100), 224(84), 223(62), 240(54) \\
239(42), 226(25)\end{array}$ & & & + & - \\
\hline \multirow[t]{2}{*}{ M1 } & $\mathrm{P}$ & 3.93 & $\mathrm{C}_{16} \mathrm{H}_{13} \mathrm{O}_{5}$ & 285.0757 & 285.0752 & -1.93 & $\begin{array}{c}\mathrm{MS}^{2}[285]: 270(100), 229(18), 225(14), 285(11) \\
170(11), 145(11) \\
\operatorname{MS}^{3}[270]: 242(100), 152(16), 214(12)\end{array}$ & & \multirow[t]{2}{*}{2.09} & + & - \\
\hline & $\mathrm{N}$ & 3.93 & $\mathrm{C}_{16} \mathrm{H}_{11} \mathrm{O}_{5}$ & 283.0600 & 283.0611 & 3.43 & $\begin{array}{l}\mathrm{MS}^{2}[283]: 268(100) \\
\mathrm{MS}^{3}[268]: 240(100)\end{array}$ & & & + & - \\
\hline M2 & $\mathrm{N}$ & 3.96 & $\begin{array}{c}\mathrm{C}_{15} \mathrm{H}_{9} \mathrm{O}_{8} \\
\mathrm{~S}\end{array}$ & 349.0012 & 349.0022 & 2.77 & $\mathrm{MS}^{2}[349]: 269(100)$ & & -0.06 & + & - \\
\hline M3 & $\mathrm{P}$ & 4.27 & $\mathrm{C}_{16} \mathrm{H}_{13} \mathrm{O}_{5}$ & 285.0757 & 285.0753 & -1.51 & $\begin{array}{c}\operatorname{MS}^{2}[285]: 179(100), 165(21) \\
\operatorname{MS}^{3}[179]: 151(100), 109(31), 111(30)\end{array}$ & & - & + & - \\
\hline \multirow{2}{*}{ M4 } & $\mathrm{P}$ & 4.38 & $\mathrm{C}_{27} \mathrm{H}_{31} \mathrm{O}_{15}$ & 595.1657 & 595.1645 & -2.11 & $\begin{array}{l}\mathrm{MS}^{2}[595]: 271(100), 433(62), 497(20) \\
\operatorname{MS}^{3}[271]: 243(100), 215(97), 153(39)\end{array}$ & & & + & - \\
\hline & $\mathrm{N}$ & 4.38 & $\mathrm{C}_{27} \mathrm{H}_{29} \mathrm{O}_{15}$ & 593.1501 & 593.1513 & 2.05 & $\operatorname{MS}^{2}[593]: 269(100), 431(14)$ & & & + & - \\
\hline
\end{tabular}


Table 1. Cont.

\begin{tabular}{|c|c|c|c|c|c|c|c|c|c|c|c|}
\hline Peak & $\begin{array}{l}\text { Ion } \\
\text { Mode }\end{array}$ & $t_{\mathrm{R}} / \mathrm{min}$ & Formula & $\begin{array}{l}\text { Theoretical } \\
\text { Mass } m / z\end{array}$ & $\begin{array}{l}\text { Experimental } \\
\text { Mass } m / z\end{array}$ & $\begin{array}{r}\text { Error } \\
(\mathrm{ppm})\end{array}$ & MS/MS Fragment Ions & Identification/Reactions & $\begin{array}{l}\text { Clog } P \\
\text { Value }\end{array}$ & $\mathbf{U}$ & $\mathbf{P}$ \\
\hline \multirow[t]{2}{*}{ M5 } & $\mathrm{P}$ & 4.47 & $\mathrm{C}_{21} \mathrm{H}_{21} \mathrm{O}_{11}$ & 449.1078 & 449.1065 & -1.36 & $\begin{array}{c}\text { MS }^{2}[449]: 287(100), 272(45), 271(32) \\
433(14), 273(13) \\
\text { MS }^{3}[287]: 187(100), 153(24), 259(3)\end{array}$ & & - & - & + \\
\hline & $\mathrm{N}$ & 4.47 & $\mathrm{C}_{21} \mathrm{H}_{19} \mathrm{O}_{11}$ & 447.0922 & 447.0927 & 1.17 & $\begin{array}{c}\mathrm{MS}^{2}[447]: 285(100) \\
\mathrm{MS}^{3}[285]: 241(100), 243(98), 217(85), 175(71) \\
\text { 199(54), 285(50), 201(40), 151(19), 133(18) }\end{array}$ & & & - & + \\
\hline \multirow{2}{*}{ M6 } & $\mathrm{P}$ & 4.56 & $\mathrm{C}_{21} \mathrm{H}_{19} \mathrm{O}_{11}$ & 447.0922 & 447.0910 & -2.59 & $\begin{array}{c}\mathrm{MS}^{2}[447]: 271(100) \\
\mathrm{MS}^{3}[271]: 153(100), 215(98), 243(75), 253(51) \\
149(38), 159(28)\end{array}$ & & -0.47 & + & + \\
\hline & $\mathrm{N}$ & 4.56 & $\mathrm{C}_{21} \mathrm{H}_{17} \mathrm{O}_{11}$ & 445.0765 & 445.0779 & 2.95 & $\begin{array}{c}\mathrm{MS}^{2}[445]: 269(100), 175(26) \\
\mathrm{MS}^{3}[269]: 269(100), 224(37), 241(27), 197(25) \\
225(21), 181(17), 227(16), 213(14)\end{array}$ & & & + & + \\
\hline M7 & $\mathrm{P}$ & 4.59 & $\mathrm{C}_{16} \mathrm{H}_{13} \mathrm{O}_{5}$ & 285.0757 & 285.0752 & -2.07 & $\begin{array}{l}\mathrm{MS}^{2}[285]: 165(100), 270(33), 191(18), 164(11) \\
\text { MS }^{3}[165]: 123(100), 137(66), 109(23), 141(20)\end{array}$ & & - & + & - \\
\hline \multirow{2}{*}{ M8 } & $\mathrm{P}$ & 4.61 & $\mathrm{C}_{15} \mathrm{H}_{11} \mathrm{O}_{5}$ & 271.0601 & 271.0595 & -2.18 & $\begin{array}{l}\left.\text { MS²}^{2} 271\right]: 153(100), 215(78), 243(67), 253(37), \\
\text { 149(29), 159(18), 145(18), 271(17), 225(11) }\end{array}$ & & & + & + \\
\hline & $\mathrm{N}$ & 4.61 & $\mathrm{C}_{15} \mathrm{H}_{9} \mathrm{O}_{5}$ & 269.0444 & 269.0453 & 3.23 & $\begin{array}{c}\mathrm{MS}^{2}[269]: 269(100), 181(57), 201(54), 225(52) \\
\text { 224(35), 197(29), 180(21) }\end{array}$ & & & + & + \\
\hline M9 & $\mathrm{N}$ & 4.64 & $\mathrm{C}_{15} \mathrm{H}_{9} \mathrm{O}_{9} \mathrm{~S}$ & 364.9961 & 364.9971 & 2.44 & $\begin{array}{c}\mathrm{MS}^{2}[365]: 285(100) \\
\mathrm{MS}^{3}[285]: 257(100), 176(56), 177(52), 243(37) \\
241(30), 229(26), 217(24)\end{array}$ & & - & + & - \\
\hline M10 & $\mathrm{N}$ & 4.85 & $\mathrm{C}_{21} \mathrm{H}_{19} \mathrm{O}_{11}$ & 447.0922 & 447.0927 & 1.03 & $\begin{array}{c}\mathrm{MS}^{2}[447]: 284(100), 285(78), 255(15), \\
\text { 270(14), 327(12) }\end{array}$ & & - & - & + \\
\hline
\end{tabular}


Table 1. Cont

\begin{tabular}{|c|c|c|c|c|c|c|c|c|c|c|c|}
\hline Peak & $\begin{array}{l}\text { Ion } \\
\text { Mode }\end{array}$ & $t_{\mathrm{R}} / \mathrm{min}$ & Formula & $\begin{array}{l}\text { Theoretical } \\
\text { Mass } m / z\end{array}$ & $\begin{array}{l}\text { Experimental } \\
\text { Mass } m / z\end{array}$ & $\begin{array}{r}\text { Error } \\
(\mathrm{ppm})\end{array}$ & MS/MS Fragment Ions & Identification/Reactions & $\begin{array}{l}\text { Clog } P \\
\text { Value }\end{array}$ & $\mathbf{U}$ & $\mathbf{P}$ \\
\hline \multirow[t]{2}{*}{ M11 } & $\mathrm{P}$ & 4.88 & $\mathrm{C}_{21} \mathrm{H}_{19} \mathrm{O}_{11}$ & 447.0922 & 447.0905 & -3.89 & $\begin{array}{c}\mathrm{MS}^{2}[447]: 271(100) \\
\mathrm{MS}^{3}[271]: 153(100), 215(95), 243(47), 149(32) \\
\text { 253(31), 145(19) }\end{array}$ & & \multirow[t]{2}{*}{0.43} & + & - \\
\hline & $\mathrm{N}$ & 4.88 & $\mathrm{C}_{21} \mathrm{H}_{17} \mathrm{O}_{11}$ & 445.0765 & 445.0780 & 3.29 & $\begin{array}{c}\mathrm{MS}^{2}[445]: 225(100), 201(63), 269(47), 181(38), \\
213(27), 197(27)\end{array}$ & & & + & + \\
\hline M12 & $\mathrm{N}$ & 5.01 & $\mathrm{C}_{21} \mathrm{H}_{19} \mathrm{O}_{10}$ & 431.0972 & 431.0988 & 3.43 & $\begin{array}{c}\operatorname{MS}^{2}[431]: 268(100), 269(30) \\
\operatorname{MS}^{3}[268]: 223(100), 224(98), 267(75), 239(47) \\
240(41), 198(35)\end{array}$ & & - & + & - \\
\hline M13 & $\mathrm{N}$ & 5.02 & $\mathrm{C}_{16} \mathrm{H}_{11} \mathrm{O}_{5}$ & 283.0600 & 283.0609 & 2.97 & $\begin{array}{l}\operatorname{MS}^{2}[283]: 268(100) \\
\operatorname{MS}^{3}[268]: 240(100)\end{array}$ & & - & + & - \\
\hline M14 & $\mathrm{N}$ & 5.11 & $\mathrm{C}_{21} \mathrm{H}_{19} \mathrm{O}_{11}$ & 447.0922 & 447.0937 & 3.29 & $\mathrm{MS}^{2}[447]: 271(100), 285(21), 175(16), 403(10)$ & & - & + & - \\
\hline M15 & $\mathrm{N}$ & 5.11 & $\mathrm{C}_{21} \mathrm{H}_{17} \mathrm{O}_{11}$ & 445.0765 & 445.0780 & 3.29 & $\begin{array}{c}\mathrm{MS}^{2}[445]: 269(100), 175(15), 153(12) \\
\mathrm{MS}^{3}[269]: 225(100), 201(55), 241(28) \\
\text { 197(24), 269(18) }\end{array}$ & & 0.43 & + & - \\
\hline M16 & $\mathrm{P}$ & 5.52 & $\mathrm{C}_{15} \mathrm{H}_{11} \mathrm{O}_{5}$ & 271.0601 & 271.0596 & -1.84 & $\begin{array}{c}\mathrm{MS}^{2}[271]: 215(100), 153(82), 243(61), 149(38) \\
253(36), 159(20), 145(14), 225(13)\end{array}$ & & - & + & - \\
\hline
\end{tabular}


Table 1. Cont.

\begin{tabular}{|c|c|c|c|c|c|c|c|c|c|c|c|}
\hline Peak & $\begin{array}{l}\text { Ion } \\
\text { Mode }\end{array}$ & $t_{\mathrm{R}} / \mathrm{min}$ & Formula & $\begin{array}{l}\text { Theoretical } \\
\text { Mass } m / z\end{array}$ & $\begin{array}{l}\text { Experimental } \\
\text { Mass } m / z\end{array}$ & $\begin{array}{r}\text { Error } \\
(\mathrm{ppm})\end{array}$ & MS/MS Fragment Ions & Identification/Reactions & $\begin{array}{l}\text { Clog } P \\
\text { Value }\end{array}$ & $\mathbf{U}$ & $\mathbf{P}$ \\
\hline M17 & $\mathrm{N}$ & 5.52 & $\mathrm{C}_{23} \mathrm{H}_{21} \mathrm{O}_{11}$ & 473.1079 & 473.1092 & 2.80 & $\mathrm{MS}^{2}[473]: 268(100), 269(66), 311(12)$ & & - & + & - \\
\hline M18 & $\mathrm{N}$ & 5.82 & $\mathrm{C}_{15} \mathrm{H}_{9} \mathrm{O}_{6}$ & 285.0393 & 285.0400 & 2.37 & $\mathrm{MS}^{2}[285]: 217(100), 199(19), 241(16), 175(10)$ & & 1.77 & + & - \\
\hline \multirow{2}{*}{ M19 } & $\mathrm{P}$ & 5.83 & $\mathrm{C}_{15} \mathrm{H}_{11} \mathrm{O}_{8} \mathrm{~S}$ & 351.0169 & 351.0162 & -1.92 & $\begin{array}{c}\mathrm{MS}^{2}[351]: 271(100), 333(21) \\
\mathrm{MS}^{3}[271]: 153(100), 253(81), 215(63), 243(50) \\
159(32), 149(22), 197(12)\end{array}$ & & \multirow{2}{*}{0.84} & + & - \\
\hline & $\mathrm{N}$ & 5.83 & $\mathrm{C}_{15} \mathrm{H}_{9} \mathrm{O}_{8} \mathrm{~S}$ & 349.0012 & 349.0023 & 2.94 & $\begin{array}{c}\mathrm{MS}^{2}[349]: 269(100) \\
\mathrm{MS}^{3}[269]: 225(100), 227(95), 175(51), 269(36) \\
241(32), 240(30), 197(24), 224(23)\end{array}$ & & & + & - \\
\hline M20 & $\mathrm{N}$ & 5.92 & $\mathrm{C}_{15} \mathrm{H}_{9} \mathrm{O}_{5}$ & 269.0444 & 269.0454 & 3.68 & $\begin{array}{c}\mathrm{MS}^{2}[269]: 225(100), 269(96), 181(71), 227(68), \\
201(54), 224(43), 197(41)\end{array}$ & & - & + & - \\
\hline M21 & $\mathrm{N}$ & 5.92 & $\mathrm{C}_{16} \mathrm{H}_{11} \mathrm{O}_{6}$ & 299.0549 & 299.0559 & 2.89 & $\begin{array}{c}\operatorname{MS}^{2}[299]: 269(100) \\
\operatorname{MS}^{3}[269]: 241(100), 224(48), 173(43), 180(22)\end{array}$ & & - & + & - \\
\hline M22 & $\mathrm{N}$ & 6.38 & $\mathrm{C}_{15} \mathrm{H}_{9} \mathrm{O}_{8} \mathrm{~S}$ & 349.0012 & 349.0018 & 1.45 & $\begin{array}{c}\operatorname{MS}^{2}[349]: 269(100) \\
\operatorname{MS}^{3}[269]: 225(100), 227(65), 269(39), 176(31) \\
\text { 197(26), 181(22), 169(21), 213(12), 241(10) }\end{array}$ & $\mathrm{HO}_{3}$ & 0.85 & + & - \\
\hline
\end{tabular}


Table 1. Cont.

\begin{tabular}{|c|c|c|c|c|c|c|c|c|c|c|c|}
\hline Peak & $\begin{array}{l}\text { Ion } \\
\text { Mode }\end{array}$ & $t_{\mathrm{R}} / \mathrm{min}$ & Formula & $\begin{array}{l}\text { Theoretical } \\
\text { Mass } m / z\end{array}$ & $\begin{array}{l}\text { Experimental } \\
\text { Mass } m / z\end{array}$ & $\begin{array}{r}\text { Error } \\
(\mathrm{ppm})\end{array}$ & MS/MS Fragment Ions & Identification/Reactions & $\begin{array}{c}\text { Clog } P \\
\text { Value }\end{array}$ & $\mathbf{U}$ & $\mathbf{P}$ \\
\hline M23 & $\mathrm{P}$ & 6.53 & $\mathrm{C}_{15} \mathrm{H}_{11} \mathrm{O}_{6}$ & 287.0550 & 287.0544 & -2.32 & $\begin{array}{l}\mathrm{MS}^{2}[287]: 272(100), 286(46), 153(44), 241(39), \\
\text { 269(34), 271(33), 231(19), 287(18), 259(13) }\end{array}$ & & 1.81 & + & - \\
\hline \multirow{2}{*}{ M24 } & $\mathrm{P}$ & 6.62 & $\mathrm{C}_{16} \mathrm{H}_{13} \mathrm{O}_{5}$ & 285.0757 & 285.0751 & -2.28 & $\begin{array}{c}\mathrm{MS}^{2}[285]: 285(100), 270(90), 229(17), 225(15) \\
\text { MS }^{3}[270]: 242(100), 152(11)\end{array}$ & & \multirow{2}{*}{2.99} & + & - \\
\hline & $\mathrm{N}$ & 6.62 & $\mathrm{C}_{16} \mathrm{H}_{11} \mathrm{O}_{5}$ & 283.0600 & 283.0610 & 3.32 & $\begin{array}{l}\text { MS²}^{2}[283]: 268(100) \\
\text { MS }^{3}[268]: 240(100)\end{array}$ & & & + & - \\
\hline M25 & $\mathrm{P}$ & 6.98 & $\mathrm{C}_{17} \mathrm{H}_{15} \mathrm{O}_{6}$ & 315.0864 & 315.0856 & -2.36 & $\begin{array}{c}\operatorname{MS}^{2}[315]: 300(100), 283(20) \\
\operatorname{MS}^{3}[300]: 167(100), 283(69), 299(42), 244(36) \\
166(32), 272(27)\end{array}$ & & - & + & - \\
\hline M26 & $\mathrm{N}$ & 7.93 & $\mathrm{C}_{12} \mathrm{H}_{9} \mathrm{O}_{3}$ & 201.0570 & 201.0553 & 3.38 & $\mathrm{MS}^{2}[201]: 173(100), 159(94), 157(75)$ & & - & + & - \\
\hline M27 & $\mathrm{P}$ & 7.97 & $\mathrm{C}_{13} \mathrm{H}_{11} \mathrm{O}_{3}$ & 215.0702 & 215.0697 & -2.65 & $\begin{array}{c}\mathrm{MS}^{2}[215]: 197(100), 169(64), 187(31), 147(18), \\
173(15), 159(10)\end{array}$ & & 2.67 & + & - \\
\hline \multirow[t]{2}{*}{ M28 } & $\mathrm{P}$ & 8.03 & $\mathrm{C}_{15} \mathrm{H}_{11} \mathrm{O}_{5}$ & 271.0601 & 271.0590 & -3.98 & $\begin{array}{c}\mathrm{MS}^{2}[271]: 271(100), 153(32), 215(25), 243(19) \\
\mathrm{MS}^{3}[271]: 153(100), 215(71), 243(58) \\
253(35), 149(25)\end{array}$ & & \multirow[t]{2}{*}{2.41} & + & + \\
\hline & $\mathrm{N}$ & 8.03 & $\mathrm{C}_{15} \mathrm{H}_{9} \mathrm{O}_{5}$ & 269.0444 & 269.0442 & -1.08 & $\begin{array}{c}\text { MS }^{2}[269]: 269(100), 225(52), 181(47), 201(44) \\
224(32), 197(26), 241(23)\end{array}$ & & & + & + \\
\hline
\end{tabular}


Table 1. Cont.

\begin{tabular}{|c|c|c|c|c|c|c|c|c|c|c|c|}
\hline Peak & $\begin{array}{l}\text { Ion } \\
\text { Mode }\end{array}$ & $t_{\mathrm{R}} / \mathrm{min}$ & Formula & $\begin{array}{l}\text { Theoretical } \\
\text { Mass } m / z\end{array}$ & $\begin{array}{l}\text { Experimental } \\
\text { Mass } m / z\end{array}$ & $\begin{array}{r}\text { Error } \\
(\mathrm{ppm})\end{array}$ & MS/MS Fragment Ions & Identification/Reactions & $\begin{array}{l}\text { Clog } P \\
\text { Value }\end{array}$ & $\mathbf{U}$ & $\mathbf{P}$ \\
\hline M29 & $\mathrm{N}$ & 8.03 & $\mathrm{C}_{14} \mathrm{H}_{9} \mathrm{O}_{3}$ & 225.0546 & 225.0546 & -0.09 & $\begin{array}{c}\mathrm{MS}^{2}[225]: 197(100), 181(98), 196(33), 169(27), \\
183(19), 180(17)\end{array}$ & & 2.49 & + & - \\
\hline M30 & $\mathrm{N}$ & 8.03 & $\mathrm{C}_{14} \mathrm{H}_{9} \mathrm{O}_{4}$ & 241.0495 & 241.0494 & -0.52 & $\begin{array}{c}\mathrm{MS}^{2}[241]: 213(100), 197(56), 199(22), 169(14) \\
196(11), 173(11)\end{array}$ & & 1.82 & + & - \\
\hline M31 & $\mathrm{N}$ & 8.03 & $\mathrm{C}_{13} \mathrm{H}_{7} \mathrm{O}_{4}$ & 227.0340 & 227.0339 & -0.07 & $\begin{array}{c}\operatorname{MS}^{2}[227]: 183(100), 199(40), 155(19) \\
66(13), 159(10)\end{array}$ & & 0.49 & + & - \\
\hline M32 & $\mathrm{N}$ & 8.14 & $\mathrm{C}_{12} \mathrm{H}_{9} \mathrm{O}_{3}$ & 201.0570 & 201.0552 & 2.78 & $\mathrm{MS}^{2}[201]: 159(100), 173(98), 133(18)$ & & - & + & - \\
\hline M33 & $\mathrm{P}$ & 8.24 & $\mathrm{C}_{16} \mathrm{H}_{13} \mathrm{O}_{6}$ & 301.0706 & 301.0700 & -2.17 & $\begin{array}{c}\operatorname{MS}^{2}[301]: 286(100) \\
\operatorname{MS}^{3}[286]: 285(100), 258(31), 286(14), 168(13)\end{array}$ & & - & + & - \\
\hline
\end{tabular}


Table 1. Cont

\begin{tabular}{|c|c|c|c|c|c|c|c|c|c|c|c|}
\hline Peak & $\begin{array}{l}\text { Ion } \\
\text { Mode }\end{array}$ & $t_{\mathrm{R}} / \mathrm{min}$ & Formula & $\begin{array}{l}\text { Theoretical } \\
\text { Mass } m / z\end{array}$ & $\begin{array}{l}\text { Experimental } \\
\text { Mass } m / z\end{array}$ & $\begin{array}{r}\text { Error } \\
(\mathrm{ppm})\end{array}$ & MS/MS Fragment Ions & Identification/Reactions & $\begin{array}{l}\text { Clog } P \\
\text { Value }\end{array}$ & $\mathbf{U}$ & $\mathbf{P}$ \\
\hline \multirow[b]{2}{*}{ M34 } & $\mathrm{P}$ & 8.46 & $\mathrm{C}_{16} \mathrm{H}_{13} \mathrm{O}_{6}$ & 301.0706 & 301.0700 & -2.37 & $\begin{array}{c}\operatorname{MS}^{2}[301]: 286(100), 269(41), 241(14) \\
\operatorname{MS}^{3}[286]: 258(100), 153(83), 285(21), 269(12)\end{array}$ & & \multirow[b]{2}{*}{2.25} & + & - \\
\hline & $\mathrm{N}$ & 8.46 & $\mathrm{C}_{16} \mathrm{H}_{11} \mathrm{O}_{6}$ & 299.0549 & 299.0559 & 2.89 & $\begin{array}{c}\mathrm{MS}^{2}[299]: 284(100) \\
\mathrm{MS}^{3}[284]: 256(100), 255(60), 227(37), 283(30) \\
212(30), 211(24), 240(20), 239(15)\end{array}$ & & & + & - \\
\hline M35 & $\mathrm{P}$ & 8.79 & $\mathrm{C}_{16} \mathrm{H}_{13} \mathrm{O}_{6}$ & 301.0706 & 301.0700 & -2.08 & $\begin{array}{c}\mathrm{MS}^{2}[301]: 286(100), 269(74), 241(25), 153(17) \\
\mathrm{MS}^{3}[286]: 153(100), 258(36), 229(21) \\
269(14), 230(10)\end{array}$ & & 2.25 & + & - \\
\hline M36 & $\mathrm{P}$ & 10.07 & $\mathrm{C}_{17} \mathrm{H}_{15} \mathrm{O}_{5}$ & 299.0903 & 299.0906 & -2.84 & $\begin{array}{c}\mathrm{MS}^{2}[299]: 284(100) \\
\mathrm{MS}^{3}[284]: 256(100), 253(53), 161(32), 269(32) \\
108(27), 228(27), 152(21)\end{array}$ & & 2.67 & + & - \\
\hline M37 & $\mathrm{P}$ & 10.28 & $\mathrm{C}_{17} \mathrm{H}_{15} \mathrm{O}_{5}$ & 299.0903 & 299.0905 & -3.08 & $\begin{array}{c}\operatorname{MS}^{2}[299]: 284(100), 243(20), 166(20), 299(13), \\
239(12), 267(11) \\
\operatorname{MS}^{3}[284]: 256(100), 166(25), 267(11) \\
255(11), 283(10)\end{array}$ & & 3.57 & + & - \\
\hline M38 & $\mathrm{N}$ & 12.34 & $\mathrm{C}_{15} \mathrm{H}_{9} \mathrm{O}_{5}$ & 269.0444 & 269.0453 & 3.23 & $\begin{array}{c}\mathrm{MS}^{2}[269]: 269(100), 181(61), 201(55), 225(53) \\
224(40), 197(33), 180(26), 183(23), 133(11)\end{array}$ & & - & + & - \\
\hline \multirow[t]{2}{*}{ M39 } & $\mathrm{P}$ & 12.44 & $\mathrm{C}_{16} \mathrm{H}_{13} \mathrm{O}_{5}$ & 285.0757 & 285.0749 & -3.02 & $\begin{array}{c}\mathrm{MS}^{2}[285]: 270(100), 229(49), 253(42), 152(31) \\
123(25), 269(19) \\
\operatorname{MS}^{3}[270]: 152(100), 269(87), 242(40) \\
253(36), 252(31)\end{array}$ & & \multirow[t]{2}{*}{2.99} & + & - \\
\hline & $\mathrm{N}$ & 12.44 & $\mathrm{C}_{16} \mathrm{H}_{11} \mathrm{O}_{5}$ & 283.0600 & 283.0609 & 2.97 & $\begin{array}{c}\mathrm{MS}^{2}[283]: 268(100) \\
\mathrm{MS}^{3}[268]: 267(100), 239(68), 224(68), 240(61) \\
223(43), 226(22), 211(11)\end{array}$ & & & + & - \\
\hline
\end{tabular}


Table 1. Cont.

\begin{tabular}{|c|c|c|c|c|c|c|c|c|c|c|c|}
\hline Peak & $\begin{array}{l}\text { Ion } \\
\text { Mode }\end{array}$ & $t_{\mathrm{R}} / \mathrm{min}$ & Formula & $\begin{array}{l}\text { Theoretical } \\
\text { Mass } m / z\end{array}$ & $\begin{array}{l}\text { Experimental } \\
\text { Mass } m / z\end{array}$ & $\begin{array}{r}\text { Error } \\
(\mathrm{ppm})\end{array}$ & MS/MS Fragment Ions & Identification/Reactions & $\begin{array}{c}\text { Clog } P \\
\text { Value }\end{array}$ & $\mathbf{U}$ & $\mathbf{P}$ \\
\hline \multirow{2}{*}{ M40 } & $\mathrm{P}$ & 3.85 & $\mathrm{C}_{15} \mathrm{H}_{11} \mathrm{O}_{4}$ & 255.0652 & 255.0647 & -1.90 & $\begin{array}{c}\text { MS }^{2}[255]: 199(100), 137(65), 227(52) \\
237(23), 145(12)\end{array}$ & & & + & + \\
\hline & $\mathrm{N}$ & 3.85 & $\mathrm{C}_{15} \mathrm{H}_{11} \mathrm{O}_{4}$ & 253.0495 & 253.0505 & 3.77 & $\begin{array}{c}\mathrm{MS}^{2}[253]: 253(100), 224(52), 209(43), 225(35) \\
\text { 197(26), 208(18), 124(14), 223(11) }\end{array}$ & & - & + & - \\
\hline \multirow[t]{2}{*}{ M41 } & $\mathrm{P}$ & 3.86 & $\mathrm{C}_{21} \mathrm{H}_{21} \mathrm{O}_{9}$ & 417.1180 & 417.1170 & -2.42 & $\begin{array}{c}\mathrm{MS}^{2}[417]: 255(100) \\
\mathrm{MS}^{3}[255]: 199(100), 227(45), 181(40) \\
137(24), 209(20)\end{array}$ & & 0.37 & + & - \\
\hline & $\mathrm{N}$ & 3.86 & $\mathrm{C}_{21} \mathrm{H}_{19} \mathrm{O}_{9}$ & 415.1023 & 415.1035 & 2.73 & $\begin{array}{c}\mathrm{MS}^{2}[415]: 253(100), \text { 252(15) } \\
\mathrm{MS}^{3}[253]: 253(100), 135(53), 160(46), 225(27)\end{array}$ & & & + & - \\
\hline \multirow[t]{2}{*}{ M42 } & $\mathrm{P}$ & 3.86 & $\mathrm{C}_{21} \mathrm{H}_{19} \mathrm{O}_{10}$ & 431.0973 & 431.0960 & -2.44 & $\begin{array}{c}\mathrm{MS}^{2}[431]: 255(100) \\
\mathrm{MS}^{3}[255]: 199(100), 137(33), 255(28), 227(19) \\
145(16), 211(10)\end{array}$ & & - & + & - \\
\hline & $\mathrm{N}$ & 3.86 & $\mathrm{C}_{21} \mathrm{H}_{17} \mathrm{O}_{10}$ & 429.0815 & 429.0830 & 3.16 & $\begin{array}{c}\mathrm{MS}^{2}[429]: 253(100), 175(41) \\
\operatorname{MS}^{3}[253]: 209(100), 253(91), 183(23)\end{array}$ & & & + & - \\
\hline M43 & $\mathrm{N}$ & 3.86 & $\mathrm{C}_{22} \mathrm{H}_{21} \mathrm{O}_{11}$ & 461.1078 & 461.1091 & 2.80 & $\begin{array}{c}\mathrm{MS}^{2}[461]: 253(100), 415(37), 298(15), \\
240(13), 284(12)\end{array}$ & & - & + & - \\
\hline \multirow[t]{2}{*}{ M44 } & $\mathrm{P}$ & 4.26 & $\mathrm{C}_{21} \mathrm{H}_{21} \mathrm{O}_{9}$ & 417.1180 & 417.1164 & -3.81 & $\begin{array}{c}\mathrm{MS}^{2}[417]: 255(100) \\
\mathrm{MS}^{3}[255]: 199(100), 237(53), 137(47) \\
\text { 227(22), 157(10) }\end{array}$ & & 0.56 & + & - \\
\hline & $\mathrm{N}$ & 4.26 & $\mathrm{C}_{21} \mathrm{H}_{19} \mathrm{O}_{9}$ & 415.1023 & 415.1035 & 2.73 & $\mathrm{MS}^{2}[415]: 253(100), 135(53), 225(27)$ & & & + & - \\
\hline M45 & $\mathrm{N}$ & 4.82 & $\mathrm{C}_{15} \mathrm{H}_{9} \mathrm{O}_{4}$ & 253.0495 & 253.0505 & 3.62 & $\begin{array}{c}\mathrm{MS}^{2}[253]: 253(100), 224(52), 225(39), 209(33) \\
197(25), 208(12)\end{array}$ & & - & + & \\
\hline
\end{tabular}


Table 1. Cont.

\begin{tabular}{|c|c|c|c|c|c|c|c|c|c|c|c|}
\hline Peak & $\begin{array}{l}\text { Ion } \\
\text { Mode }\end{array}$ & $t_{\mathrm{R}} / \mathrm{min}$ & Formula & $\begin{array}{l}\text { Theoretical } \\
\text { Mass } m / z\end{array}$ & $\begin{array}{l}\text { Experimental } \\
\text { Mass } m / z\end{array}$ & $\begin{array}{r}\text { Error } \\
(\mathrm{ppm})\end{array}$ & MS/MS Fragment Ions & Identification/Reactions & $\begin{array}{l}\text { Clog } P \\
\text { Value }\end{array}$ & $\mathbf{U}$ & $\mathbf{P}$ \\
\hline \multirow{2}{*}{ M46 } & $\mathrm{P}$ & 4.88 & $\mathrm{C}_{15} \mathrm{H}_{11} \mathrm{O}_{7} \mathrm{~S}$ & 335.0220 & 335.0208 & -3.55 & $\begin{array}{c}\mathrm{MS}^{2}[335]: 255(100), 316(44) \\
\mathrm{MS}^{3}[255]: 199(100), 137(33), 255(28), 227(19) \\
145(16), 211(10)\end{array}$ & \multirow[t]{2}{*}{$\mathrm{HO}^{\mathrm{O}} \mathrm{O} \quad \mathrm{SO}_{3}$} & \multirow[t]{2}{*}{-} & + & - \\
\hline & $\mathrm{N}$ & 4.88 & $\mathrm{C}_{15} \mathrm{H}_{9} \mathrm{O}_{7} \mathrm{~S}$ & 333.0063 & 333.0071 & 0.79 & $\begin{array}{c}\mathrm{MS}^{2}[333]: 253(100) \\
\mathrm{MS}^{3}[253]: 211(100), 209(95), 225(91) \\
\text { 224(56), 135(42) }\end{array}$ & & & + & - \\
\hline M47 & $\mathrm{N}$ & 6.31 & $\mathrm{C}_{16} \mathrm{H}_{11} \mathrm{O}_{6}$ & 299.0549 & 299.0549 & -0.48 & $\begin{array}{c}\mathrm{MS}^{2}[299]: 281(100), 163(79), 253(39), 237(28), \\
225(13), 224(11)\end{array}$ & & - & + & - \\
\hline \multirow[b]{2}{*}{ M48 } & $\mathrm{P}$ & 6.33 & $\mathrm{C}_{15} \mathrm{H}_{11} \mathrm{O}_{4}$ & 255.0652 & 255.0648 & -1.67 & $\begin{array}{c}\mathrm{MS}^{2}[255]: 255(100), 199(18), 137(13) \\
\text { MS }^{3}[255]: 199(100), 137(97), 227(42), 171(12)\end{array}$ & & \multirow[b]{2}{*}{2.08} & + & + \\
\hline & $\mathrm{N}$ & 6.33 & $\mathrm{C}_{15} \mathrm{H}_{9} \mathrm{O}_{4}$ & 253.0495 & 253.0499 & 1.56 & $\begin{array}{c}\mathrm{MS}^{2}[253]: 253(100) \\
\mathrm{MS}^{3}[253]: 253(100), 225(53), 209(46) \\
197(18), 208(17)\end{array}$ & & & + & + \\
\hline M49 & $\mathrm{P}$ & 6.34 & $\mathrm{C}_{14} \mathrm{H}_{11} \mathrm{O}_{3}$ & 227.0703 & 227.0698 & -2.16 & $\begin{array}{c}\mathrm{MS}^{2}[227]: 182(100), 199(47), 184(26), \\
157(22), 86(22)\end{array}$ & & 3.26 & + & - \\
\hline M50 & $\mathrm{P}$ & 9.92 & $\mathrm{C}_{16} \mathrm{H}_{13} \mathrm{O}_{4}$ & 269.0803 & 269.0801 & -2.58 & $\operatorname{MS}^{2}[269]: 254(100), 237(46), 213(37), 253(12)$ & & - & + & - \\
\hline M51 & $\mathrm{N}$ & 3.49 & $\mathrm{C}_{21} \mathrm{H}_{19} \mathrm{O}_{9}$ & 415.1023 & 415.1036 & 2.94 & $\begin{array}{l}\operatorname{MS}^{2}[415]: 295(100) \\
\operatorname{MS}^{3}[295]: 267(100)\end{array}$ & & 0.02 & + & - \\
\hline
\end{tabular}


Table 1. Cont.

\begin{tabular}{|c|c|c|c|c|c|c|c|c|c|c|c|}
\hline Peak & $\begin{array}{l}\text { Ion } \\
\text { Mode }\end{array}$ & $t_{\mathrm{R}} / \mathrm{min}$ & Formula & $\begin{array}{l}\text { Theoretical } \\
\text { Mass } m / z\end{array}$ & $\begin{array}{l}\text { Experimental } \\
\text { Mass } m / z\end{array}$ & $\begin{array}{r}\text { Error } \\
(\mathrm{ppm})\end{array}$ & MS/MS Fragment Ions & Identification/Reactions & $\begin{array}{l}\text { Clog } P \\
\text { Value }\end{array}$ & $\mathbf{U}$ & $\mathbf{P}$ \\
\hline M52 & $\mathrm{P}$ & 4.16 & $\mathrm{C}_{16} \mathrm{H}_{15} \mathrm{O}_{5}$ & 287.0915 & 287.0908 & -2.23 & $\begin{array}{c}\mathrm{MS}^{2}[287]: 259(100), 66(30), 231(18), \\
241(18), 213(14)\end{array}$ & & - & + & - \\
\hline M53 & $\mathrm{P}$ & 5.13 & $\mathrm{C}_{15} \mathrm{H}_{13} \mathrm{O}_{5}$ & 273.0758 & 273.0751 & -2.27 & $\begin{array}{c}\mathrm{MS}^{2}[273]: 255(100), 66(85), 179(82) \\
123(58), 245(31)\end{array}$ & & - & + & - \\
\hline M54 & $\mathrm{P}$ & 5.19 & $\mathrm{C}_{16} \mathrm{H}_{15} \mathrm{O}_{6}$ & 303.0863 & 303.0870 & 2.39 & $\begin{array}{c}\mathrm{MS}^{2}[303]: 230(100), 285(87), 217(30) \\
\text { 257(22), } 272(14)\end{array}$ & & - & + & - \\
\hline M55 & $\mathrm{P}$ & 6.72 & $\mathrm{C}_{16} \mathrm{H}_{15} \mathrm{O}_{5}$ & 287.0915 & 287.0906 & -2.75 & $\begin{array}{c}\mathrm{MS}^{2}[287]: 272(100), 286(40), 66(38) \\
270(36), 193(29)\end{array}$ & & - & + & - \\
\hline \multirow{2}{*}{ M56 } & $\mathrm{P}$ & 7.64 & $\mathrm{C}_{15} \mathrm{H}_{13} \mathrm{O}_{5}$ & 273.0758 & 273.0751 & -2.49 & $\begin{array}{c}\text { MS }^{2}[273]: 255(100), 179(84), 123(42), 151(37) \\
107(10), 245(10)\end{array}$ & & & + & - \\
\hline & $\mathrm{N}$ & 7.64 & $\mathrm{C}_{15} \mathrm{H}_{11} \mathrm{O}_{5}$ & 271.0601 & 271.0609 & 3.10 & $\begin{array}{c}\mathrm{MS}^{2}[271]: 165 \text { (100), } \mathrm{MS}^{3}[165]: 137(100), \\
93(26), 109(25), 121(24)\end{array}$ & & & + & - \\
\hline M57 & $\mathrm{P}$ & 8.03 & $\mathrm{C}_{15} \mathrm{H}_{13} \mathrm{O}_{5}$ & 273.0758 & 273.0750 & -2.86 & $\begin{array}{c}\mathrm{MS}^{2}[273]: 272(100), 216(49), 244(41), 153(41) \\
154(37), 254(24)\end{array}$ & & - & + & - \\
\hline
\end{tabular}


Table 1. Cont

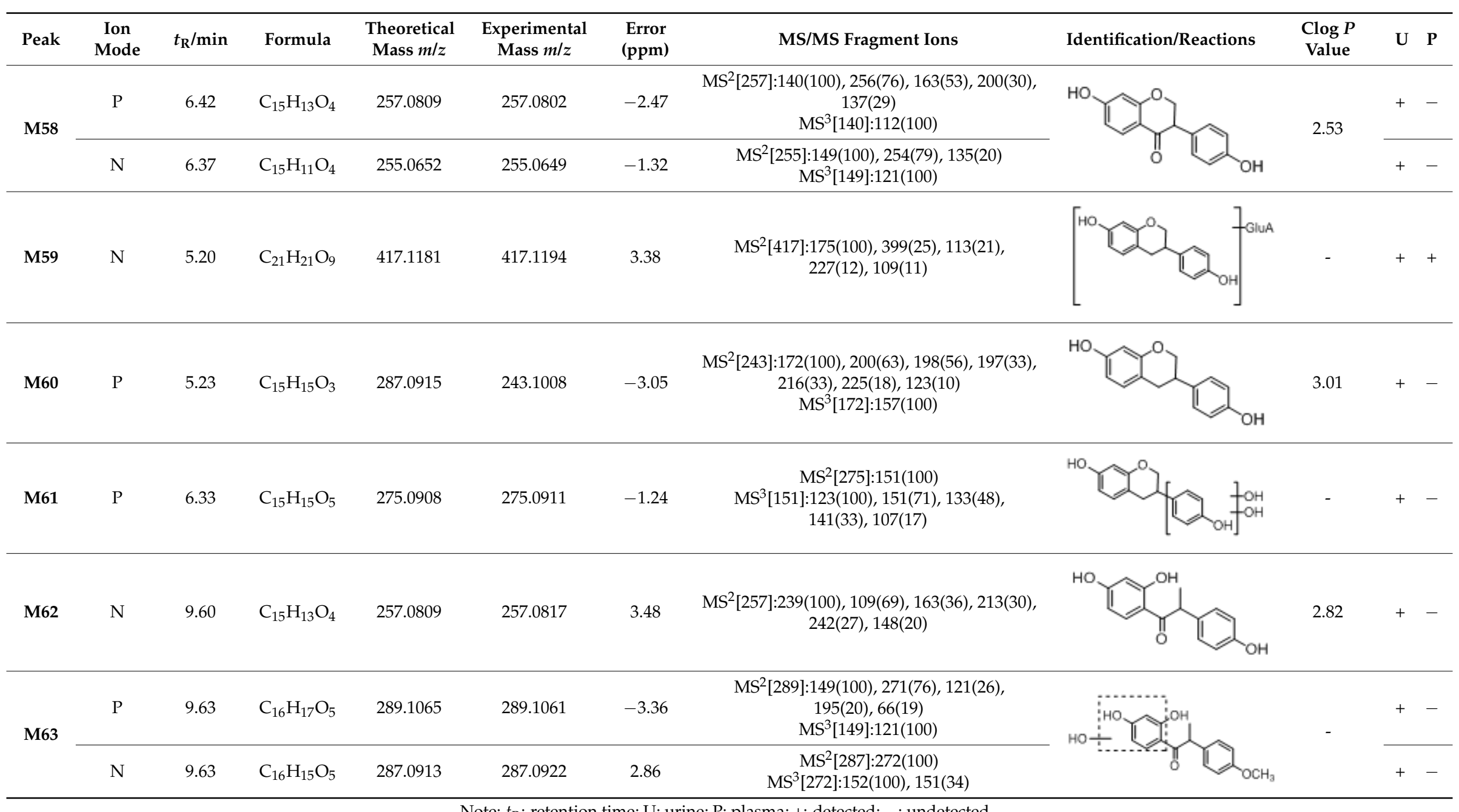

Note: $t_{\mathrm{R}}$ : retention time; U: urine; P: plasma; +: detected; -: undetected. 


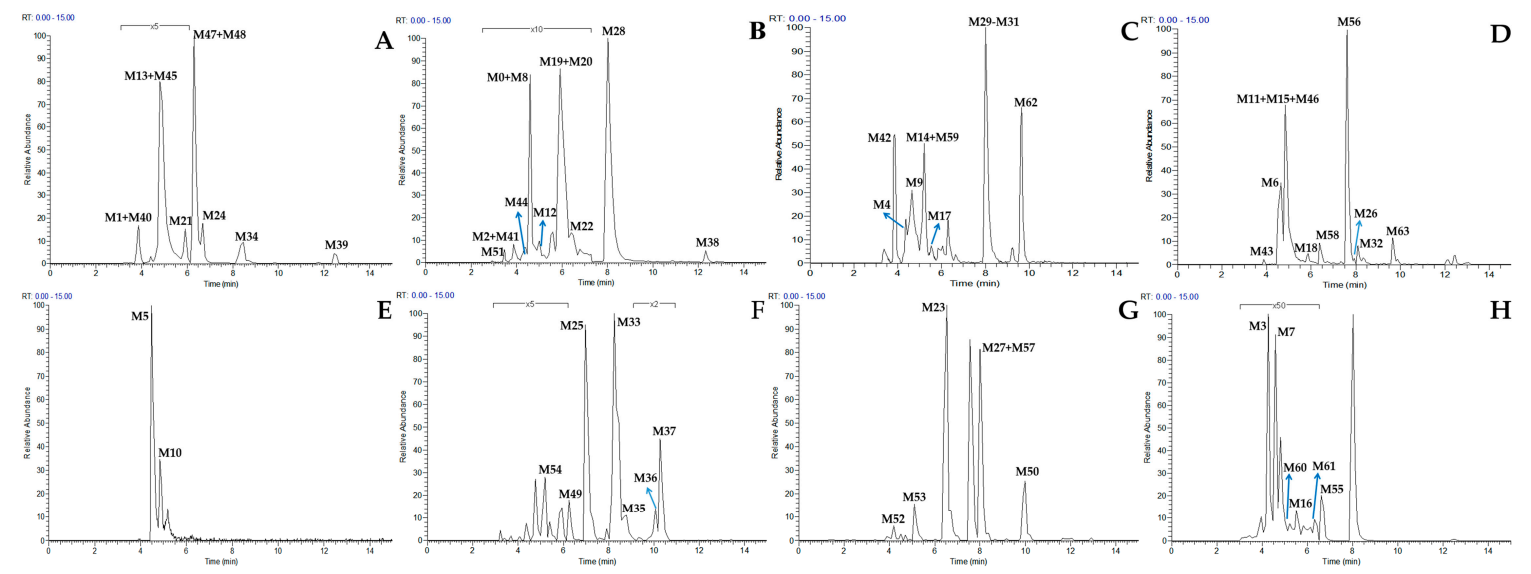

Figure 6. High resolution extracted ion chromatograms of genistin metabolites ((A-E) for negative ion mode and (F-H) for positive ion mode): (A) $m / z$ 253.0495, 283.0600, 299.0549, 447.0922; (B) $m / z$ 269.0444, 349.0012, 415.1023, 431.0972; (C) $m / z$ 225.0546, 227.0340, 241.0495, 257.0809, 364.9961, 417.1181, 429.0815, 447.0922, 473.1079, 593.1501; (D) $m / z$ 201.0570, 255.0652, 271.0601, 285.0393, 287.0913, 333.0063, 445.0765, 461.1078; (E) $m / z$ 447.0922; (F) $m / z$ 227.0703, 299.0903, 301.0706, 303.0863, 315.0864; (G) $m / z$ 215.0702, 269.0803, 273.0758, 287.0550, 287.0915; (H) $m / z$ 243.1016, 271.0601, 275.0908, 285.0757, 287.0915.

M4, eluted at $4.38 \mathrm{~min}$, gave rise to its $[\mathrm{M}+\mathrm{H}]^{+}$ion at $m / z 595.1645\left(\mathrm{C}_{27} \mathrm{H}_{31} \mathrm{O}_{15},-2.11 \mathrm{ppm}\right)$. It was $162 \mathrm{Da}$ higher than that of genistin, indicating that it might be glucosylation product of genistin. In its ESI-MS ${ }^{2}$ spectrum, successive NLFs of $162 \mathrm{Da}(m / z 595 \rightarrow m / z 433 \rightarrow m / z$ 271) were observed, which demonstrated that the glucosyl group was probably introduced into the glucose moiety.

M17, eluted at $5.52 \mathrm{~min}$, possessed the experimental $[\mathrm{M}-\mathrm{H}]^{-}$ion at $m / z 473.1092\left(\mathrm{C}_{23} \mathrm{H}_{21} \mathrm{O}_{11}\right.$, $2.80 \mathrm{ppm}$ ), which was $42 \mathrm{Da}$ higher than that of genistin, meaning that it might be an acetylation product of genistin. The ion at $m / z 311$ was observed by the NLF of $162 \mathrm{Da}$ and the ion at $m / z 269$ was also found by successive losing the NLFs of $162 \mathrm{Da}$ and $42 \mathrm{Da}$, which further confirmed our deduction.

Metabolites M8, M16, M20, M28, and M38 showed their retention times at 4.61, 5.52, 5.92, 8.03 , and $12.34 \mathrm{~min}$, respectively, were exhibited the same theoretical $[\mathrm{M}+\mathrm{H}]^{+}$ion at $m / z 271.0601$ $\left(\mathrm{C}_{15} \mathrm{H}_{11} \mathrm{O}_{5}\right.$, error within $\left.\pm 4.00 \mathrm{ppm}\right)$ or the same theoretical $[\mathrm{M}-\mathrm{H}]^{-}$ion at $m / z 269.0444\left(\mathrm{C}_{15} \mathrm{H}_{9} \mathrm{O}_{5}\right.$, error within $\pm 4.00 \mathrm{ppm})$. Among them, M28 was determined as genistein by comparing the retention time and fragmentation behaviors with genistein reference standard. M8, M16, M20, and M38 possessed the similar characteristic fragments (such as $m / z 153, m / z 215, m / z 243, m / z 253$, and $m / z 225$ in positive ion mode and $m / z 181, m / z 201, m / z 225$, and $m / z 197$ in negative ion mode) with M28 and therefore they were preliminarily identified as the isomers of genistein under the present situation.

Metabolites M1, M3, M7, M13, M24, and M39 with a protonated $[\mathbf{M}+\mathrm{H}]^{+}$ion at $m / z 285.0757$ $\left(\mathrm{C}_{16} \mathrm{H}_{13} \mathrm{O}_{5}\right.$, error within $\left.\pm 3.50 \mathrm{ppm}\right)$ or a deprotonated $[\mathrm{M}-\mathrm{H}]^{-}$ion at $m / z 283.0600\left(\mathrm{C}_{16} \mathrm{H}_{11} \mathrm{O}_{5}\right.$, error within $\pm 3.50 \mathrm{ppm}$ ), were individually eluted at 3.93, 4.27, 4.59, 5.02, 6.62, and $12.44 \mathrm{~min}$. They were $14 \mathrm{Da}$ more than genistein whether in positive ion mode or in negative ion mode. The DPI at $m / z 270\left[\mathrm{M}+\mathrm{H}-\mathrm{CH}_{3}\right]^{+}$indicated that M1, M24, and M39 might be methylated products of genistein. The DPI at $m / z 242$ generated by the NLF of CO and the DPI at $m / z 152$ yielded by RDA rearrangement occurred on positions 1 and 3 further confirmed our conjecture. Due to a compound with a larger $C \log P$ value would have a longer retention time in a reverse phase (RP) chromatographic system [34]. Therefore M1, M24, and M39 were putatively thought as 5-O-methylgenistein (Clog $P$, 2.09), 4'-O-methylgenistein ( $\mathrm{Clog} P, 2.99)$, and 7-O-methyl-genistein $(\mathrm{Clog} P, 2.99)$.

There was a NLF of $15 \mathrm{Da}\left(\mathrm{CH}_{3}\right)$ from M13, indicating that a methyl group existed in M13. The subsequent $\mathrm{MS}^{3}$ of $m / z 268$ generated the fragment ion at $m / z 240$ by the NLF of 28 Da. According the limited information, M13 was guessed as the O-methyl product of an isomer of 
genistein. Different from the above methylated products, the ion of losing $15 \mathrm{Da}$ was not found in the mass spectrum of M3 and M7. As a result, we considered they might be the C-methylgenistein. The DPI at $m / z 109$ yielded by RDA rearrangement occurred on positions 10 and 4 further confirmed our conjecture.

M36 and M37, possessed experimental protonated molecular ions $[\mathrm{M}+\mathrm{H}]^{+}$at $m / z 299.0906$ $\left(\mathrm{C}_{17} \mathrm{H}_{15} \mathrm{O}_{5},-2.84 \mathrm{ppm}\right)$ and $m / z 299.0905\left(\mathrm{C}_{17} \mathrm{H}_{15} \mathrm{O}_{5},-3.08 \mathrm{ppm}\right)$, were detected at 10.07 and $10.28 \mathrm{~min}$, respectively. They were $14 \mathrm{Da}$ higher than that of methylgenistein, so they were could be the dimethylation products of genistein. The sequential NLFs of $15 \mathrm{Da}(\mathrm{m} / z 299 \rightarrow m / z 284 \rightarrow m / z 269)$ occurred in ESI-MS ${ }^{n}$ spectra, coupling with the DPIs at $m / z 152$ and $m / z 166$ (generated by the RDA rearrangement occurred on positions 1 and 3) further validated that one methyl group was added to A-ring, while the other methyl was introduced to B-ring. They were tentatively interpreted as 5,4'-dimethygenistein and 7, $4^{\prime}$-dimethygenistein.

M6, M11, and M15 with their retention times of 4.56, 4.88, and $5.11 \mathrm{~min}$ separately, were $176 \mathrm{Da}$ higher than that of genistein. They exhibited the same molecular ion at $m / z 445.0765\left(\left[\mathrm{C}_{21} \mathrm{H}_{17} \mathrm{O}_{11}\right]^{-}\right.$, error within $\pm 3.50 \mathrm{ppm})$ and showed the NLF of $176 \mathrm{Da}(\mathrm{m} / z 445 \rightarrow m / z 269)$ in their ESI-MS ${ }^{2}$ spectra, which indicated the presence of glucuronidation group. Meanwhile, the mass fragmentation behaviors of $m / z 269$ were similar to those of genistein, which provided adequate evidence that they were genistein-O-glucuronides. Due to the structure of genistein, there were three sites that might accomodate glucose aldehyde acidification. So M6, M11, and M15 were provisionally characterized as 5-O-glucuronide-genistein, 7-O-glucuronide-genistein, and 4'-O-glucuronide-genistein based on their Clog $P$ values.

Metabolites M2, M19, and M22, whose elution times were 3.96, 5.83, and 6.38 min, were $80 \mathrm{Da}$ higher than that of genistein in negative ion mode, which indicated the occurrence of sulfonation reaction. In its MS/MS² spectrum, the DPI at $m / z 269$ was detected due to the NLF of 80 Da from the parent ion at $m / z 349$ and in its MS/MS spectrum, the similar characteristic fragments with genistein were observed, which all indicated that the three metabolites were the sulfonation products of genistein. According to their $C \log P$ values, they were briefly inferred to be 5-O-sulfate-genistein, $4^{\prime}$-O-sulfate-genistein, and 7-O-sulfate-genistein, respectively.

Metabolites M18 and M23 were eluted at 5.82 and $6.53 \mathrm{~min}$, respectively, and produced the same theoretical $[\mathrm{M}-\mathrm{H}]^{-}$ion at $m / z 285.0393\left(\mathrm{C}_{15} \mathrm{H}_{9} \mathrm{O}_{6}\right.$, error within $\left.\pm 2.50 \mathrm{ppm}\right)$ in negative ion mode, which were $16 \mathrm{Da}$ higher than that of genistein. Thus they were supposed to be hydroxylation products of genistein due to the similar fragmentation behaviors. The DPI at $m / z 153$ (RDA rearrangement occurred on positions 1 and 3 of C-ring) of M23 in positive ion mode, indicating that the hydroxyl group was added to the B-ring, which was deduced as 3'-hydroxygenistein [35]. M18 was provisionally characterized as 8-hydroxygenistein on the basis of the calculated $C \log P$ values [36]. M9, possessed the same fragment ions $(m / z 285,257,229$, and 217) with $\mathbf{M} 23$ in negative ion mode, was 80 Da more than that of M23, manifesting the presence of sulfuric group.

M5, M10, and M14, whose retention times are 4.47, 4.85, and $5.11 \mathrm{~min}$, were $162 \mathrm{Da}$ more than that of hydroxylation of genistein, indicating they might be glucosylation products of hydroxylated genistein. The DPI at $m / z 285\left([\mathrm{M}-\mathrm{H}-\mathrm{Glc}]^{-}\right)$was yielded by a NLF of glucose moiety, which identified our hypothesis. Therefore, they were putatively identified as hydroxygenistein-O-glucoside.

M21, M33, M34, and M35 were eluted at 5.92, 6.31, 8.24, 8.46, and 8.79 min, respectively. M21 possessed a deprotonated molecule ion at $m / z 299.0549\left(\mathrm{C}_{16} \mathrm{H}_{11} \mathrm{O}_{6}, 2.89 \mathrm{ppm}\right)$ and M33, M34, and M35 showed a protonated molecule ion at $m / z 301.0706\left(\mathrm{C}_{16} \mathrm{H}_{13} \mathrm{O}_{6}\right.$, error within $\left.\pm 2.50 \mathrm{ppm}\right)$. The NLF of $30 \mathrm{Da}\left(\mathrm{CH}_{3} \mathrm{O}\right)$ was observed in the ESI-MS ${ }^{2}$ spectra of M21, which indicated that it was a product of $O$-methoxygenistein. In the ESI-MS ${ }^{3}$ spectra, the similar characteristic ions were tested with genistein, further confirming our speculation. The DPIs of M33, M34, and M35 were $m / z 286$ by loss of $15 \mathrm{Da}\left(\mathrm{CH}_{3}\right)$ and $m / z 258$ via a NLF of $28 \mathrm{Da}(\mathrm{CO})$, suggesting that they were the methylation of hydroxylated genistein. 
The ion at $m / z 153$ of M34 and M35 were produced by RDA rearrangement occurred on positions 1 and 3 of $\mathrm{C}$-ring, suggesting that hydroxylation and methylation were occurred on B-ring. Therefore they were identified as $3^{\prime}$-hydroxyl-4'-O-methylgenistein and $3^{\prime}$-methoxylgenistein, respectively. The fragment ion at $m / z 168$ of $\mathbf{M} 33$ indicated that hydroxyl was attributed to A-ring.

M25, 14 Da more than that of M34, presented a protonated molecule ion at $m / z 315.0856$ $\left(\mathrm{C}_{17} \mathrm{H}_{15} \mathrm{O}_{6},-2.36 \mathrm{ppm}\right)$ with the retention time of $6.98 \mathrm{~min}$. The DPI at $\mathrm{m} / z 300$ with the loss of $\mathrm{CH}_{3}$ and $m / z 167$ produced by the RDA rearrangement occurred on positions 1 and 3 provided the evidence for identifying the metabolite. In addition, the fragmentation behaviour was similar with M34. Hence we deemed M25 was the methylated product of M34 with the methyl group attaching to A-ring.

M26, M27, and M29-M32 were the cleavage products of genistein by losing different groups. The metabolite M27 with the quasi-molecular ion at $m / z 215.0697\left(\mathrm{C}_{13} \mathrm{H}_{11} \mathrm{O}_{3},-2.65 \mathrm{ppm}\right)$ was discovered at $7.97 \mathrm{~min}$ in the ESI-MS ${ }^{1}$ spectra. In the $\mathrm{MS}^{2}$, the base peak of $\mathrm{m} / z 197$ was generated by loss of $\mathrm{C}_{2} \mathrm{H}_{2} \mathrm{O}_{3}\left(2 \mathrm{CO}+\mathrm{H}_{2} \mathrm{O}\right)$. Based on the possible metabolic path, its structure was suspected as showed in Table 1. In negative ion mode, $\mathbf{M} 26$ and $\mathbf{M} 32$ showed the $[\mathrm{M}-\mathrm{H}]^{-}$at $m / z 201.0553$ $\left(\mathrm{C}_{12} \mathrm{H}_{9} \mathrm{O}_{3}, 3.38 \mathrm{ppm}\right)$ and $201.0552\left(\mathrm{C}_{12} \mathrm{H}_{9} \mathrm{O}_{3}, 2.78 \mathrm{ppm}\right)$, respectively. The characteristic fragment ions were $m / z 173,159,157$, and 133 by loss of $\mathrm{CO}, \mathrm{C}_{2} \mathrm{H}_{2} \mathrm{O}, \mathrm{CO}_{2}$, and $\mathrm{C}_{4} \mathrm{H}_{4} \mathrm{O}$. According to the above data, both compounds were decided as showed in Table 1 .

M29 $\left(\mathrm{C}_{14} \mathrm{H}_{9} \mathrm{O}_{3}\right)$ and $\mathbf{M} 30\left(\mathrm{C}_{14} \mathrm{H}_{9} \mathrm{O}_{4}\right)$ were generated via the loss of $\mathrm{CO}_{2}$ and $\mathrm{CO}$ at ring $\mathrm{C}$, yielding $\left[\mathrm{M}-\mathrm{H}-\mathrm{CO}_{2}\right](\mathrm{m} / z 225.0546,-0.09 \mathrm{ppm})$ and $[\mathrm{M}-\mathrm{H}-\mathrm{CO}](\mathrm{m} / z$ 241.0494, $-0.52 \mathrm{ppm}$ ), respectively. Both ions possessed stable structures with a basic phenanthrene framework. The same fragmentation behaviour with genistein in negative ion mode supported our speculation. Different from M29 and M30, M31 was formed by the loss of $\mathrm{C}_{2} \mathrm{H}_{2} \mathrm{O}$ occurred at ring A, which was confirmed by its further fragmentation behavior with losing $\mathrm{CO}_{2}, \mathrm{CO}$, and $\mathrm{CO}+\mathrm{CO}_{2}$. Therefore, the structures were showed in Table 1.

\subsubsection{Identification of Metabolites with the Templates of Daidzin and Daidzein}

M40, M45, and M48 gave a deprotonated ion at $m / z 253.0495\left(\mathrm{C}_{15} \mathrm{H}_{9} \mathrm{O}_{4}\right.$, error within $\left.\pm 4.00 \mathrm{ppm}\right)$, which were eluted at $3.85,4.82$, and $6.33 \mathrm{~min}$, respectively. By comparing the chromatographic retention time and MS/MS spectra, M48 was positively identified as daidzein. Due to the similar fragmentation behaviours with M48, we conjectured M40 and M45 were the isomerides of daidzein.

M41 and M44 were 162 Da higher than daidzein, indicating that they were the glucoside conjugate of daidzein. In the $\mathrm{MS}^{2}$, one fragment ion, $m / z 255$, was formed by the loss of the glucose moiety from the precursor ion. In the $\mathrm{MS}^{3}$, the neutral losses of $\mathrm{CO}, 2 \mathrm{CO}$, and $\mathrm{C}_{8} \mathrm{H}_{6} \mathrm{O}$ from $m / z 255$ were observed and the corresponding fragment ions at $m / z 227,199$, and 137 were generated, respectively. Based on the reference compound, M41 was hopefully identified as daidzin, therefore M44 was deduced as $4^{\prime}$-glucoside-daidzein and the calculated Clog $P$ value further confirmed our guess. M43, showed the $[\mathrm{M}-\mathrm{H}]^{-}$ion at $m / z 461.1091\left(\mathrm{C}_{22} \mathrm{H}_{21} \mathrm{O}_{11}, 2.80 \mathrm{ppm}\right)$, was 46 Da more than daidzin and the highest product ion at $m / z 253$ was found in the mass spectrum of $\mathrm{MS}^{2}$. The second highest product ion at $\mathrm{m} / z 415$ was formed via the loss of $\mathrm{CH}_{2} \mathrm{O}_{2}$. Hence we suspected the structure of $\mathbf{M} 43$ as showed in Table 1.

M42 exhibited a protonated ion at $m / z 431.0973\left(\mathrm{C}_{21} \mathrm{H}_{19} \mathrm{O}_{10},-2.44 \mathrm{ppm}\right)$, which was $176 \mathrm{Da}$ higher than that of daidzein. $\mathrm{MS}^{2}$ gave the base peak of $m / z 255$ via loss of a glucuronide moiety (176 Da) from $m / z$ 431. In the MS ${ }^{3}$ spectrum of $\mathbf{M} 42$, the $m / z 255$ ion yielded specific ions at $m / z$ 199, 137,227 , and 145 due to the loss of $2 \mathrm{CO}, \mathrm{C}_{8} \mathrm{H}_{6} \mathrm{O}, \mathrm{CO}$, and $\mathrm{C}_{6} \mathrm{H}_{6} \mathrm{O}_{2}$. Therefore $\mathbf{M} 42$ was supposed as the daidzein-O-glucuronide. The $\mathrm{MS}^{2}$ of $\mathrm{M} 46\left([\mathrm{M}+\mathrm{H}]^{+}, m / z\right.$ 335.0208) generated the fragment ion at $m / z 255$ by the NLF of $80 \mathrm{Da}$ and the fragmentation behaviour of $m / z 255$ was similar with daidzein by losing $2 \mathrm{CO}, \mathrm{C}_{8} \mathrm{H}_{6} \mathrm{O}, \mathrm{CO}$, and $\mathrm{C}_{6} \mathrm{H}_{6} \mathrm{O}_{2}$, so we proposed that $\mathbf{M} 46$ was $\mathrm{O}$-sulfate-daidzein.

M50, eluted at $9.92 \mathrm{~min}$, was $14 \mathrm{Da}$ higher than daidzein in positive ion mode, manifesting that it might be a methylated product of daidzein. The DPIs at $m / z 254$ (100\%), 237 (46\%), and 213 (37\%) 
were generated by the NLFs of $\mathrm{CH}_{3}, \mathrm{CH}_{4} \mathrm{O}\left(\mathrm{H}_{2} \mathrm{O}+\mathrm{CH}_{2}\right)$, and $2 \mathrm{CO}$. Because of two active sites existed in daidzein, $\mathbf{M} 50$ was putatively appraised as $\mathrm{O}$-methyldaidzein.

M47 was eluted at 6.31 min with a deprotonated $[\mathrm{M}-\mathrm{H}]^{-}$ion at $m / z 299.0549\left(\mathrm{C}_{16} \mathrm{H}_{11} \mathrm{O}_{6}\right.$, $-0.48 \mathrm{ppm}$ ). It was $46 \mathrm{Da}$ higher than that of daidzein, showing that there was a $\mathrm{CH}_{2} \mathrm{O}_{2}$ more than daidzein in molecular composition. The DPIs at $m / z 253,225$, and 224 offered the evidence to support our guess. The ion at $m / z 163$ was formed by RDA rearrangement which occurred on positions 0 and 3 on C-ring, which indicated that the $\mathrm{CH}_{2} \mathrm{O}$ was attached to B-ring as shown in Table 1 [36].

Metabolite M49 with a protonated $[\mathrm{M}+\mathrm{H}]^{+}$ion at $m / z 227.0698\left(\mathrm{C}_{14} \mathrm{H}_{11} \mathrm{O}_{3},-2.16 \mathrm{ppm}\right)$, was eluted at $6.34 \mathrm{~min}$. The DPI at $m / z 199$ was found in the mass spectrum of $\mathrm{MS}^{2}$ by the NLF of $\mathrm{CO}$ with no other characteristic ions observed. So it was putatively identified as the decarbonylation product of daidzein.

\subsubsection{Identification of Metabolites with the Templates of Puerarin}

M51, possessed the deprotonated $[\mathrm{M}-\mathrm{H}]^{-}$ion at $415.1036\left(\mathrm{C}_{21} \mathrm{H}_{19} \mathrm{O}_{9}, 2.94 \mathrm{ppm}\right)$, was $162 \mathrm{Da}$ more than that of daidzein with the retention time of $3.49 \mathrm{~min}$. It was definitely identified as puerarin by comparing with the information of the standard.

\subsubsection{Identification of Metabolites with the Template of Hydrogenated Genistein}

M53, M56, and M57 were detected at 5.13, 7.64, and 8.03 min and possessed the same $[\mathrm{M}+\mathrm{H}]^{+}$ ion at $m / z 273.0758\left(\mathrm{C}_{15} \mathrm{H}_{13} \mathrm{O}_{5}\right.$, error within $\left.\pm 3.00 \mathrm{ppm}\right)$, which were $2 \mathrm{Da}$ higher than that of genistein. In their ESI-MS ${ }^{2}$ spectra, DPIs of M53 and M56 at $m / z 255\left(\left[\mathrm{M}+\mathrm{H}-\mathrm{H}_{2} \mathrm{O}\right]^{+}\right)$and $m / z 245$ $\left(\left[\mathrm{M}+\mathrm{H}-\mathrm{CO}^{+}\right)\right.$were found and $m / z 153$ of $\mathbf{M} 57$ was also detected via RDA rearrangement occurred on positions 1 and 3 . So we suspected that they were hydrogenated products of genistein and its isomers.

The two compounds of M52 and M55 showed $[\mathrm{M}+\mathrm{H}]^{+}$at $m / z 287.0915\left(\mathrm{C}_{16} \mathrm{H}_{15} \mathrm{O}_{5}\right.$, error within $\pm 2.80 \mathrm{ppm})$, respectively, with 14 Da more than that of hydrogenated genistein. The quasi-molecular ion at $m / z 287$ presented the base peak at $m / z 259$ via the loss of $28 \mathrm{Da}$, while the base peak of M55 at $m / z 272$ was produced via a loss of $15 \mathrm{Da}$, so we deduced that M52 and M55 were the C-methylated product and $\mathrm{O}$-methylated product of genistein, respectively. M54, yielding the accurate $[\mathrm{M}+\mathrm{H}]^{+}$ at $m / z$ 303.0870, was 30 Da more than that of hydrogenated genistein. The product ions at $m / z 285$ $(87 \%)$ and $257(22 \%)$ were generated by the loss of $\mathrm{H}_{2} \mathrm{O}$ and $\mathrm{H}_{2} \mathrm{O}+\mathrm{CO}$. There was no other more information, so M54 was putatively considered as a product of $C$-methyl-hydroxylation of genistein.

\subsubsection{Identification of Metabolites with the Template of Hydrogenated Daidzein}

M58 was 2 Da higher than that of daidzein, which indicated that it might be hydrogenation product of daidzein. The most abundant fragment at $m / z 149$ in the spectra of M58 in negative ion mode was a result of RDA reaction occurred on the positions 2 and 3 . The DPI at $m / z 135$ was generated by RDA rearrangement occurred between positions 1 and 3, further conforming our conjecture.

\subsubsection{Identification of Metabolites with the Template of Equol}

M60, eluted at $5.23 \mathrm{~min}$, gave rise to its $[\mathrm{M}+\mathrm{H}]^{+}$ion at $m / z 243.1008\left(\mathrm{C}_{15} \mathrm{H}_{15} \mathrm{O}_{3},-3.05 \mathrm{ppm}\right)$. The observed DPI of $m / z 123$ was generated by RDA rearrangement which occurred on positions 1 and 3 on C-ring. Therefore, M60 was putatively identified as equol [37].

M61 was 32 Da more than that of M60, indicating M61 might be the dihydroxylated product of M60. In the ESI-MS ${ }^{2}$ spectra, the corresponding base peak at $m / z 151$ was generated by losing $124 \mathrm{Da}$ $\left(\mathrm{C}_{6} \mathrm{H}_{4} \mathrm{O}_{3}\right)$. The fragmentation ions of the subsequent $\mathrm{MS}^{3}$ of $m / z 151$ were similar with equol [37], which was consistent with our guess.

M59, possessing deprotonated $[\mathrm{M}-\mathrm{H}]^{-}$ion at $417.1194\left(\mathrm{C}_{21} \mathrm{H}_{21} \mathrm{O}_{9}, 3.38 \mathrm{ppm}\right)$, was eluted at $5.20 \mathrm{~min}$, which was $176 \mathrm{Da}$ higher than M60. Due to there was no other characteristic ions, M59 was guessed as the product of glucose aldehyde acidification of equol. 
2.4.7. Identification of Metabolites with the Template of $O$-Desmethylangolensin

M62 possessed the $[\mathrm{M}-\mathrm{H}]^{-}$ion at $m / z 257.0817\left(\mathrm{C}_{15} \mathrm{H}_{13} \mathrm{O}_{4}, 3.48 \mathrm{ppm}\right)$ with the retention time at $9.60 \mathrm{~min}$. It was $4 \mathrm{Da}$ more than that of daidzein, which indicated that it might be a dihydrogenation product of daidzein. In its ESI-MS ${ }^{2}$ spectrum, DPIs at $m / z 163\left(\left[\mathrm{M}-\mathrm{H}-\mathrm{C}_{6} \mathrm{H}_{6} \mathrm{O}\right]^{-}\right.$, produced by RDA rearrangement occurred on positions 3 and $\left.1^{\prime}\right)$ and $m / z 109\left(\left[\mathrm{M}-\mathrm{H}-\mathrm{C}_{9} \mathrm{H}_{8} \mathrm{O}_{2}\right]^{-}\right.$, formed by RDA rearrangement occurred on positions 4 and 10) indicated it should be $O$-desmethylangolensin.

M63, eluted at $9.63 \mathrm{~min}$, gave rise to its $[\mathrm{M}-\mathrm{H}]^{-}$ion at $\mathrm{m} / z 287.0922\left(\mathrm{C}_{16} \mathrm{H}_{15} \mathrm{O}_{5}, 2.86 \mathrm{ppm}\right)$. In the $\mathrm{MS}^{2}$, the loss of $\mathrm{CH}_{3}$ formed the characteristic ion at $m / z$ 272. Then the $\mathrm{MS}^{3}$ of $m / z 272$ presented the specific fragment ion at $m / z 151$ via RDA rearrangement occurred on positions 1 and 3 . According to the possible metabolic path, the methyl group was attached to $4^{\prime}-\mathrm{OH}$ to get the hydroxylation and methylation product of $\mathbf{M} 62$.

\subsection{Proposed Metabolic Pathways of Genistin-}

In our study, a total of 64 metabolites (parent drug included) were found in rats after oral administration of genistin. The proposed metabolic pathways of genistin are illustrated in Figure 7 . There was a series of metabolic reactions including methylation, hydrogenation, hydroxylation, glucosylation, glucuronidation, sulfonation, acetylation, ring cleavage and their composite reactions in vivo biotransformation. In addition, it should be noted that some cracked ring and rearrangement products were produced, such as M26, M27, M29-M32, and M49. Furthermore some special products were detected which some methyl groups were introduced to carbon rings, such as M3 and M7.

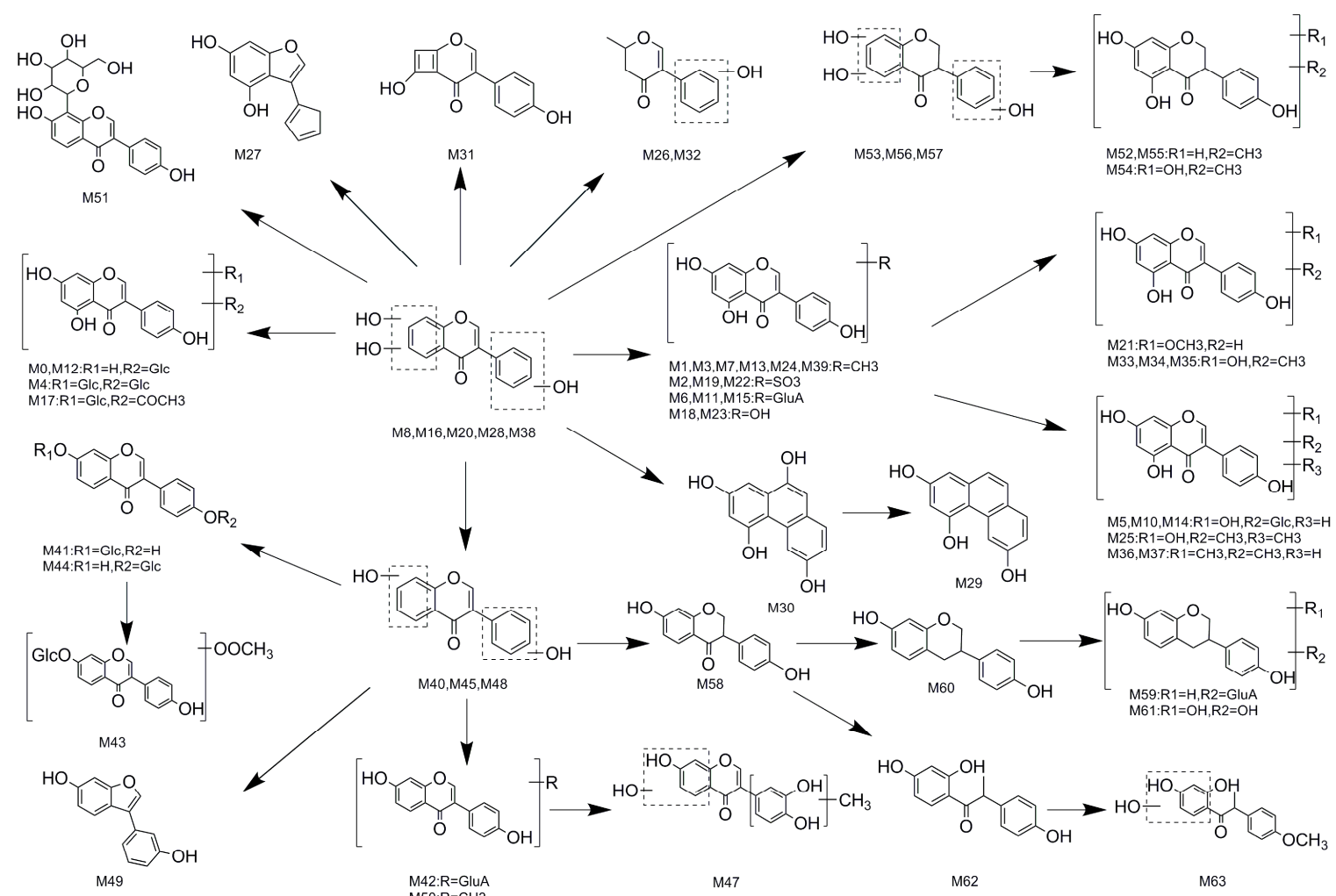

Figure 7. The proposed genistin metabolic patterns in vivo. 


\section{Materials and Methods}

\subsection{Chemicals and Reagents}

Genistin, genistein, daidzin, daidzein, and puerain were commercially provided by Chengdu Must Biotechnology Co., Ltd. (Chengdu, Sichuan, China). These five reference standards with the purity higher than 98\% were applicable to UHPLC-LTQ-Orbitrap analysis. Acetonitrile, methanol, and formic acid (HPLC grade) used in the mobile phase were obtained from Fisher Scientific (Fair Lawn, NJ, USA). In addition, the other reagents and solvents met the requirements of analytical experiments, which were from Beijing Chemical Works (Beijing, China). The ultrapure water was derived from Milli-Q Gradient $\AA 10$ water purification system (Millipore, Billerica, MA, USA). Grace Pure ${ }^{\mathrm{TM}}$ SPE C18-Low solid-phase extraction (SPE) cartridges (200 mg/3 mL, $59 \mu \mathrm{m}, 70 \AA$ ) for solid phase pretreatment of biological samples were supplied by Grace Davison Discovery Science ${ }^{\mathrm{TM}}$ (Deerfield, IL, USA).

\subsection{Animals and Drug Administration}

Eight SD rats (male, 200-240 g) were purchased from Beijing Weitong Lihua Experimental Animals Company (Beijing, China) and kept under controlled environmental conditions (temperature: $22-26^{\circ} \mathrm{C}$, relative humidity: $65-75 \%$, day and night alternation time: 12-h light/dark cycle) with free food intake and water consumption. After one week of acclimatization, rats were randomly divided into two groups ( $n=4$ each): Drug Group and Control Group. The standard of genistin was suspended in $0.5 \%$ sodium carboxymethyl cellulose (CMC-Na) solution. The Drug Group was orally administered genistin $(350 \mathrm{mg} / \mathrm{kg}$ ), while Control Group was given equivalent $0.5 \% \mathrm{CMC}-\mathrm{Na}$ solution by oral gavage. Before the experiment, all the rats were fasted for $12 \mathrm{~h}$ but free drinking water. All procedures were conducted according to the guidelines of Animal Care and Use Committee of Beijing University of Chinese Medicine and Guide for the Care and Use of Laboratory Animals of the US National Institutes of Health.

\subsection{Sample Collection and Preparation}

\subsubsection{Plasma Sample Collection}

After oral administration, the blood samples were taken from the suborbital venous plexus of the rats in Drug Group at 0.5, 1, 1.5, 2, and $4 \mathrm{~h}$ with blood volume of about $0.5 \mathrm{~mL}$. The obtained blood samples were placed in the anticoagulant EP tubes of heparin sodium. After resting for $10 \mathrm{~min}$, each blood sample was centrifuged for $15 \mathrm{~min}\left(3500 \mathrm{rpm}, 4{ }^{\circ} \mathrm{C}\right)$ and all the blood supernatants in Drug Group were merged into a collective one to get the test plasma containing the drug. The blank plasma sample was from Control Group and the method of collection was the same as the test samples. The above plasma samples were stored at $-80^{\circ} \mathrm{C}$.

\subsubsection{Urine Sample Collection}

Urine samples from 0 to $24 \mathrm{~h}$ of each rat in Drug Group were collected by using separate metabolic cages after administration. Each urine sample was centrifuged for $15 \mathrm{~min}\left(3500 \mathrm{rpm}, 4{ }^{\circ} \mathrm{C}\right)$ and all the urine supernatants in Drug Group were mixed to obtain urine test sample. The blank urine was from Control Group and the method of collection was the same as the test samples. The above urine samples were stored at $-80^{\circ} \mathrm{C}$.

Finally we used the SPE method to pretreat all biological samples, which was a method for precipitation and concentration of protein and solid residues. The SPE cartridges were pre-activated with $5 \mathrm{~mL}$ of methanol and $5 \mathrm{~mL}$ of deionized water and then $1 \mathrm{~mL}$ of plasma and urine samples were added. At last the cartridges were eluted by $5 \mathrm{~mL}$ of deionized water and $3 \mathrm{~mL}$ of methanol orderly. The methanol eluent was collected and dried under $\mathrm{N}_{2}$ at room temperature. The residue was then redissolved in $80 \mu \mathrm{L} 10 \%$ acetonitrile solution and centrifuged for $30 \mathrm{~min}\left(13,500 \mathrm{rpm}, 4^{\circ} \mathrm{C}\right)$. The supernatant was used for subsequent analysis. 


\subsection{Instruments and Analytical Conditions}

An UHPLC-LTQ-Orbitrap mass spectrometer (Thermo Scientific, Bremen, Germany) coupled with an ESI source was used to identify metabolites. The separation of samples was performed on a Waters ACQUITY BEH C18 column $(2.1 \mathrm{~mm} \times 100 \mathrm{~mm}$ i.d., $1.7 \mu \mathrm{m}$; Waters Corporation, Milford, MA, USA). The mobile phase consisted of $0.1 \%$ formic acid aqueous solution (A) and acetonitrile (B), and the linear gradient procedure was described as follows: $0-2 \mathrm{~min}, 5-20 \% \mathrm{~B} ; 2-27 \mathrm{~min}, 20-85 \% \mathrm{~B}$; $27-30 \mathrm{~min}, 85 \% \mathrm{~B}$. The column temperature was set at $30{ }^{\circ} \mathrm{C}$, and the flow rate was $0.3 \mathrm{~mL} / \mathrm{min}$ with $2 \mu \mathrm{L}$ of the injection volume.

The optimized operating parameters were set as follows: capillary temperature, $350{ }^{\circ} \mathrm{C}$; electrospray voltage, $3.5 \mathrm{kV}$; sheath gas, $35 \mathrm{arb}$; auxiliary gas, $10 \mathrm{arb}$; and probe heater temperature, $300{ }^{\circ} \mathrm{C}$. The metabolites were detected by full-scan mass analysis from $m / z$ 100-1000 with a resolution of 30,000 under positive ion and negative ion modes. The $\mathrm{MS}^{2}$ and $\mathrm{MS}^{3}$ analysis were based on the data dependent scan, and the three most abundant ions from the precursor list were selected for collision induced dissociation (CID). The collision energy was adjusted to $40 \%$ of maximum to minimize the total analysis time. The dynamic exclusion (DE) was used to prevent duplication. The repeat count was set at 5 and the dynamic repeat time was $30 \mathrm{~s}$ with the dynamic exclusion duration at $60 \mathrm{~s}$. Furthermore, $\mathrm{MS}^{\mathrm{n}}$ stages of the obtained data sets were obtained by the parent ion list (PIL)-DE dependent acquisition mode.

\subsection{Data Processing}

The collected data sets were recorded and processed by Thermo Xcalibur 2.1 workstation (Thermo Scientific, Bremen, Germany). In order to acquire as many fragment ions as possible, we selected the peaks with intensity over 10,000 under negative ion mode and 40,000 under positive ion mode to identify the metabolites. Based on the accurate mass and considering the potential element compositions and the occurrence of possible reactions, the types and number of the predicted atoms were set as follows: $\mathrm{C}$ [0-35], $\mathrm{H}$ [0-50], $\mathrm{O}$ [0-25], $\mathrm{S}$ [0-2], $\mathrm{N}$ [0-3], and ring double bond (RDB) equivalent value [0-15]. The maximum mass errors between the measured and the calculated values were fixed within $5 \mathrm{ppm}$.

\section{Conclusions}

In the present study, a single oral administration of genistin to SD rats was used to study the metabolic profile of genistin in urine and plasma both in positive and negative ion modes. Different from the previous researches, the multiple metabolite templates were set up to assist in data processing combining with DPIs and calculated $C \log P$ values. First, based on the ten metabolite templates, 101 metabolites including the prototype drug were found and confirmed positively or ambiguously according to the fragmentation patterns, accurate mass measurements, chromatographic retention times and relevant drug biotransformation knowledge. Among them, 47 metabolites were found according to the templates of genistin and genistein, which was the same as the previous research method. After using the other metabolite templates, many more metabolites were added, among which 24 metabolites were detected with the templates of daidzin and daidzein. Taking puerarin as a template, only one metabolite was found in vivo. There were also six metabolites on the basis of the template of dihydrodaidzein. Moreover, 16 metabolites with the template of hydrogenated genistein (including dihydrogenistein and tetrahydrogenistein), three metabolites based on the template of equol and four metabolites with $O$-desmethylangolensin as the template were identified respectively. After deleting the same metabolites, a total of 64 metabolites (including the parent drug) were left. The metabolite templates and the number of corresponding metabolites were as follows: genistin and genistein ( 40 metabolites), daidzin and daidzein (11 metabolites), puerarin (one metabolite), dihydrodaidzein (one metabolite), hydrogenated genistein (six metabolites), equol (three metabolites), and $O$-desmethylangolensin (two metabolites). The results demonstrated 
that genistin mainly underwent methylation, hydrogenation, hydroxylation, glucuronide conjugation, sulfate conjugation, acetylation, ring-cleavage and their composite reactions in vivo biotransformation. In conclusion, our research not only revealed the metabolites of genistein in vivo roundly, but also proposed an integrated strategy based on multiple metabolite templates to screen and identify the metabolites of natural compounds roundly. Meanwhile, an effective method based on the multiple metabolite templates combined with UHPLC-LTQ-Orbitrap mass spectrometer was established for screening metabolites.

Author Contributions: J.Z. conceived and designed the experiments; Z.W. (Zhibin Wang) supervised the experimental plan; W.Z. and Z.W. (Zijian Wang) performed the experiments; Y.L. and C.W. analyzed the data; Y.L. wrote the paper; J.Z. and Z.W. (Zhibin Wang) reviewed the manuscript; all authors read and approved the final manuscript.

Funding: This work was supported by the Beijing Nova Program (No. Z171100001117029) and the Open Project Program of Shanxi Key Laboratory of Chinese Medicine Encephalopathy (Grant No. CME-OP-2017002).

Conflicts of Interest: The authors declare no conflict of interest.

\section{References}

1. Lee, C.H.; Yang, L.; Xu, J.Z.; Venus, Y.S.Y.; Huang, Y.; Chen, Z.Y. Relative antioxidant activity of soybean isoflavones and their glycosides. Food Chem. 2005, 90, 735-741. [CrossRef]

2. Rekha, C.R.; Vijayalakshmi, G. Influence of natural coagulants on isoflavones and antioxidant activity of tofu. J. Food Sci. Technol. 2010, 47, 387-393. [CrossRef] [PubMed]

3. Villares, A.; Rostagno, M.A.; Garcia-Lafuente, A.; Guillamon, E.; Martinez, J.A. Content and profile of isoflavones in soy-based foods as a function of the production process. Food Bioprocess Technol. 2009, 4, 27-38. [CrossRef]

4. Zhao, J.H.; Arao, Y.; Sun, S.J.; Kikuchi, A.; Kayama, F. Oral administration of soy-derived genistin suppresses lipopolysaccharide-induced acute liver inflammation but does not induce thymic atrophy in the rat. Life Sci. 2006, 78, 812-819. [CrossRef] [PubMed]

5. Spagnuolo, C.; Russo, G.L.; Orhan, I.E.; Habtemariam, S.; Daglia, M.; Sureda, A.; Nabavi, S.F.; Devi, K.P.; Loizzo, M.R.; Tundis, R.; et al. Genistein and cancer: Current status, challenges, and future directions. Adv. Nutr. 2015, 6, 408-419. [CrossRef] [PubMed]

6. Gu, M.; Zheng, A.B.; Jin, J.; Cui, Y.; Zhang, N.; Che, Z.P.; Wang, Y.; Zhan, J.; Tu, W.J. Cardioprotective Effects of Genistin in Rat Myocardial Ischemia-Reperfusion Injury Studies by Regulation of P2X7/NF-kB Pathway. Evid. Based Complement. Altern. Med. 2016, 2016, 5381290. [CrossRef] [PubMed]

7. Russo, A.; Cardile, V.; Lombardo, L.; Vanella, L.; Acquaviva, R. Genistin inhibits UV light-induced plasmid DNA damage and cell growth in human melanoma cells. J. Nutr. Biochem. 2006, 17, 103-108. [CrossRef] [PubMed]

8. Xu, M.L.; Bi, C.W.; Kong, A.Y.; Dong, T.T.; Wong, Y.H.; Tsim, K.W. Flavonoids induce the expression of acetylcholinesterase in cultured osteoblasts. Chem. Biol. Interact. 2016, 259, 295-300. [CrossRef] [PubMed]

9. Klein, C.B.; King, A.A. Genistein genotoxicity: Critical considerations of in vitro exposure dose. Toxicol. Appl. Pharmacol. 2007, 224, 1-11. [CrossRef] [PubMed]

10. Touny, L.H.; Banerjee, P.P. Identification of both Myt-1 and Wee-1 as necessary mediators of the p21-independent inactivation of the Cdc-2/cyclin B1 complex and growth inhibition of TRAMP cancer cells by genistein. Prostate 2006, 66, 1542-1555. [CrossRef] [PubMed]

11. Snyder, R.D.; Gillies, P.J. Reduction of genistein clastogenicity in Chinese hamster V79 cells by daidzein and other flavonoids. Food Chem. Toxicol. 2003, 41, 1291-1298. [CrossRef]

12. Ramos, S. Effects of dietary flavonoids on apoptotic pathways related to cancer chemoprevention. J. Nutr. Biochem. 2007, 18, 427-442. [CrossRef] [PubMed]

13. Meena, R.; Supriya, C.; Pratap, R.K.; Sreenivasula, R.P. Altered spermatogenesis, steroidogenesis and suppressed fertility in adult male rats exposed to genistein, a non-steroidal phytoestrogen during embryonic development. Food Chem. Toxicol. 2017, 99, 70-77. [CrossRef] [PubMed] 
14. Zhang, Y.; Ren, Y.; Jiao, J.; Li, D.; Zhang, Y. Ultra high-performance liquid chromatography-tandem mass spectrometry for the simultaneous analysis of asparagine, sugars, and acrylamide in Maillard Reactions. Anal. Chem. 2011, 83, 3297-3304. [CrossRef] [PubMed]

15. Ma, Y.L.; Qin, H.L.; Liu, W.J.; Peng, J.Y.; Huang, L.; Zhao, X.P.; Cheng, Y.Y. Ultra-high performance liquid chromatography-mass spectrometry for the metabolomic analysis of urine in colorectal cancer. Dig. Dis. Sci. 2009, 54, 2655-2662. [CrossRef] [PubMed]

16. Grata, E.; Guillarme, D.; Glauser, G.; Boccard, J.; Carrupt, P.A.; Veuthey, J.L.; Rudaz, S.; Wolfender, J.L. Metabolite profiling of plant extracts by ultrahigh-pressure liquid chromatography at elevated temperature coupled to time-of-flight mass spectrometry. J. Chromatogr. 2009, 1216, 5660-5668. [CrossRef] [PubMed]

17. Soldi, M.; Cuomo, A.; Bonaldi, T. Improved bottom-up strategy to efficiently separate hypermodified histone peptides through ultra-HPLC separation on a bench top Orbitrap instrument. Proteomics 2014, 14, 2212-2225. [CrossRef] [PubMed]

18. Jia, W.; Ling, Y.; Lin, Y.; Chang, J.; Chu, X. Analysis of additives in dairy products by liquid chromatography coupled to quadrupole-orbitrap mass spectrometry. J. Chromatogr. A 2014, 1336, 67-75. [CrossRef] [PubMed]

19. Fornelli, L.; Ayoub, D.; Aizikov, K.; Beck, A.; Tsybin, Y.O. Middle-down analysis of monoclonal antibodies with electron transfer dissociation orbitrap fourier transform mass spectrometry. Anal. Chem. 2014, 86, 3005-3012. [CrossRef] [PubMed]

20. Wang, F.; Cao, G.S.; Li, Y.; Xu, L.L.; Wang, Z.B.; Liu, Y.; Lu, J.Q.; Zhang, J.Y. Characterization of forsythoside A metabolites in rats by a combination of UHPLC-LTQ-Orbitrap mass spectrometer with multiple data processing techniques. Biomed. Chromatogr. 2018, 32, e4164. [CrossRef] [PubMed]

21. Shang, Z.P.; Wang, F.; Dai, S.Y.; Lu, J.Q.; Wu, X.D.; Zhang, J.Y. Profiling and identification of (-)-epicatechin metabolites in rats using ultra-high performance liquid chromatography coupled with linear trap-Orbitrap mass spectrometer. Drug Test Anal. 2017, 9, 1224-1235. [CrossRef] [PubMed]

22. Tchoumtchoua, J.; Njamen, D.; Mbanya, J.C.; Skaltsounis, A.L.; Halabalaki, M. Structure-oriented UHPLC-LTQ Orbitrap-based approach as a dereplication strategy for the identification of isoflavonoids from Amphimas pterocarpoides crude extract. J. Mass Spectrom. 2013, 48, 561-575. [CrossRef] [PubMed]

23. Yan, B.; Deng, Y.; Hou, J.; Bi, Q.; Yang, M.; Jiang, B.; Liu, X.; Wu, W.; Guo, D. UHPLC-LTQ-Orbitrap MS combined with spike-in method for plasma metabonomics analysis of acute myocardial ischemia rats and pretreatment effect of Danqi Tongmai tablet. Mol. Biosyst. 2015, 11, 486-496. [CrossRef] [PubMed]

24. Shang, Z.P.; Cai, W.; Cao, Y.F.; Wang, F.; Wang, Z.B.; Lu, J.Q.; Zhang, J.Y. An integrated strategy for rapid discovery and identification of the sequential piperine metabolites in rats using ultra high-performance liquid chromatography/high resolution mass spectrometery. J. Pharm. Biomed. Anal. 2017, 146, 387-401. [CrossRef] [PubMed]

25. Chen, J.F.; Song, Y.L.; Guo, X.Y.; Tu, P.F.; Jiang, Y. Characterization of the herb-derived components in rats following oral administration of Carthamus tinctorius extract by extracting diagnostic fragment ions (DFIs) in the MSn chromatograms. Analyst 2014, 139, 6474-6485. [CrossRef] [PubMed]

26. Li, Y.F.; Ren, Q.; Jin, Y.; Wu, C.S.; Wang, C.H.; Jia, Z.X.; Zhang, J.L. Metabolic studies of four soy isoflavones in rats by HPLC-HR-MS. J. Asian Nat. Prod. Res. 2014, 16, 497-510. [CrossRef] [PubMed]

27. Hosoda, K.; Furuta, T.; Yokokawa, A.; Ogura, K.; Hiratsuka, A.; Ishii, K. Plasma profiling of intact isoflavone metabolites by high-performance liquid chromatography and mass spectrometric identification of flavone glycosides daidzin and genistin in human plasma after administration of kinako. Drug Metab. Dispos. 2008, 36, 1485-1495. [CrossRef] [PubMed]

28. Hosoda, K.; Furuta, T.; Ishii, K. Metabolism and disposition of isoflavone conjugated metabolites in humans after ingestion of kinako. Drug Metab. Dispos. 2011, 39, 1762-1767. [CrossRef] [PubMed]

29. Hosoda, K.; Furuta, T.; Ishii, K. Simultaneous determination of glucuronic acid and sulfuric acid conjugated metabolites of daidzein and genistein in human plasma by high-performance liquid chromatography. J. Chromatogr. B 2010, 878, 628-636. [CrossRef] [PubMed]

30. March, R.E.; Miao, X.S.; Metcalfe, C.D.; Stobiecki, M.; Marczak, L. A fragmentation study of an isoflavone glycoside, genistein-7-O-glucoside, using electrospray quadrupole time-of-flight mass spectrometry at high mass resolution. Int. J. Mass Spectrom. 2004, 232, 171-183. [CrossRef]

31. Kang, J.; Hick, L.A.; Price, W.E. A fragmentation study of isoflavones in negative electrospray ionization by MSn ion trap mass spectrometry and triple quadrupole mass spectrometry. Rapid Commun. Mass Spectrom. 2007, 21, 857-868. [CrossRef] [PubMed] 
32. Li, Y.H.; Dai, H.X.; Li, X.R.; Wang, L.J.; Xue, M. Analysis Fragment Pathway of Puerarin by Electron Spray Ionization Mass Spectrometry. J. Chin. Mass Spectrom. Soc. 2007, 28, 224-228.

33. Shang, Z.P.; Xin, Q.; Zhao, W.J.; Wang, Z.; Li, Q.; Zhang, J.; Cong, W. Rapid profiling and identification of puerarin metabolites in rat urine and plasma after oral administration by UHPLC-LTQ-Orbitrap mass spectrometer. J. Chromatogr. B 2017, 1068-1069, 180-192. [CrossRef] [PubMed]

34. Yao, D.; Wang, Y.; Huo, C.; Wu, Y.; Zhang, M.; Li, L.; Shi, Q.; Kiyota, H.; Shi, X. Study on the metabolites of isoalantolactone in vivo and in vitro by ultra performance liquid chromatography combined with Triple TOF mass spectrometry. Food Chem. 2017, 214, 328-338. [CrossRef] [PubMed]

35. Pandey, B.P.; Lee, N.; Choi, K.Y.; Jung, E.; Jeong, D.H.; Kim, B.G. Screening of bacterial cytochrome P450s responsible for regiospecific hydroxylation of (iso)flavonoids. Enzyme Microb. Technol. 2011, 48, 386-392. [CrossRef] [PubMed]

36. Kulling, S.E.; Honig, D.M.; Simat, T.J. Oxidative in vitro metabolism of the soy phytoestrogens daidzein and genistein. J. Agric. Food Chem. 2000, 48, 4963-4972. [CrossRef] [PubMed]

37. Muthyala, R.S.; Ju, Y.H.; Sheng, S.; Williams, L.D.; Doerge, D.R.; Katzenellenbogen, B.; Helferich, W.G.; Katzenellenbogen, J.A. Equol, a natural estrogenic metabolite from soy isoflavones: Convenient preparation and resolution of $\mathrm{R}$ - and S-equols and their differing binding and biological activity through estrogen receptors alpha and beta. Bioorgan. Med. Chem. 2004, 12, 1559-1567. [CrossRef] [PubMed]

Sample Availability: Samples of the compounds are not available from the authors. 